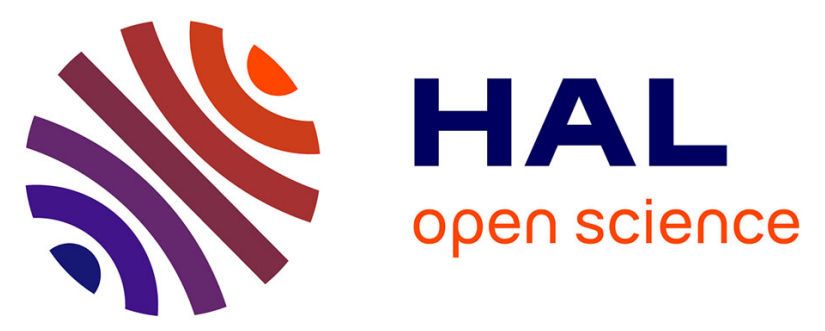

\title{
Synthèse sur le comportement et la modélisation viscoplastique d'un acier inoxydable 17-12 SPH aux températures intermédiaires $; 20 \leq \mathrm{T} \leq 700^{\circ} \mathrm{C}$
}

\author{
P. Delobelle
}

\section{- To cite this version:}

P. Delobelle. Synthèse sur le comportement et la modélisation viscoplastique d'un acier inoxydable 17-12 SPH aux températures intermédiaires; $20 \leq \mathrm{T} \leq 700^{\circ} \mathrm{C}$. Revue de Physique Appliquée, 1990, 25 (10), pp.977-999. 10.1051/rphysap:019900025010097700 . jpa-00246273

\section{HAL Id: jpa-00246273 https://hal.science/jpa-00246273}

Submitted on 1 Jan 1990

HAL is a multi-disciplinary open access archive for the deposit and dissemination of scientific research documents, whether they are published or not. The documents may come from teaching and research institutions in France or abroad, or from public or private research centers.
L'archive ouverte pluridisciplinaire HAL, est destinée au dépôt et à la diffusion de documents scientifiques de niveau recherche, publiés ou non, émanant des établissements d'enseignement et de recherche français ou étrangers, des laboratoires publics ou privés. 
Classification

Physics Abstracts

$46.30 \mathrm{~J}-62.20 \mathrm{~F}-81.40 \mathrm{~L}$

\title{
Synthèse sur le comportement et la modélisation viscoplastique d'un acier inoxydable 17-12 SPH aux températures intermédiaires ; $20 \leqslant T \leqslant 700{ }^{\circ} \mathrm{C}$
}

\section{P. Delobelle}

Laboratoire de Mécanique Appliquée - URA 279 CNRS, Faculté des Sciences et des Techniques, 16 Route de Gray, 25030 Besançon Cedex, France

(Reçu le 6 octobre 1989, révisé le 17 mai 1990, accepté le 3 juillet 1990)

\begin{abstract}
Résumé. - On montre, dans le présent article, la diversité des propriétés mécaniques monotones, cycliques et visqueuses d'un acier inoxydable austénitique 17-12 SPH sur le domaine des températures intermédiaires, $20 \leqslant T \leqslant 700{ }^{\circ} \mathrm{C}$. On met clairement en évidence des phénomènes d'interactions fortes entre dislocations et configurations de défauts ponctuels. L'incorporation de la modélisation phénoménologique de ces interactions, dans un modèle viscoplastique unifié développé par ailleurs et présentement étendu au domaine des températures étudiées, permet d'appréhender la plupart des phénomènes observés.
\end{abstract}

\begin{abstract}
This article shows the diversity of monotonic, cyclic and viscous mechanical properties of an austenitic 17-12 SPH stainless steel in the intermediate temperature range : $20 \leqslant T \leqslant 700{ }^{\circ} \mathrm{C}$. The phenomena of strong interactions between dislocations and point defect configurations are enlightened. The incorporation of the phenomenological modelization of these interactions into a unified viscoplastic model developed elsewhere and extended to the studied temperatures, allows most of the observed phenomena to be accounted for.
\end{abstract}

\section{Introduction.}

Les matériaux industriels utilisés dans certains composants d'installations modernes (industrie nucléaire dans le cas présent) sont souvent soumis à des régimes de sollicitations mécaniques et thermiques. La connaissance du comportement et le développement de lois anisothermes s'avèrent être indispensables pour la prévision de la durée de vie de ces installations. Cependant, avant de réaliser et de modéliser de vrais essais anisothermes, où sollicitations thermiques et mécaniques évoluent cycliquement et simultanément, l'identification et la modélisation phénoménologique, pour différentes isothermes, des mécanismes physiques prenant part à la déformation, sont des points incontournables. Dans le présent article, on présente les résultats relatifs à cette première étape, dans le cas d'un acier inoxydable austénitique 17-12 Mo SPH.

Bien que le comportement mécanique de cet acier (voisin du $316 \mathrm{~L}$ ) a été très étudié à température ambiante [1-3] et au voisinage de $600{ }^{\circ} \mathrm{C}$ [4-9], paradoxalement, très peu de travaux ont été réalisés de façon systématique entre ces deux températures [10-14], qui correspondent sensiblement à l'amplitude thermique maximale du cycle arrêt-fonctionnement des échangeurs dans la filière surgénérateur $\left(20-525^{\circ} \mathrm{C}\right)$.

On pallie cette lacune en présentant un ensemble assez complet de résultats expérimentaux relatifs aux propriétés thermomécaniques monotones, cycliques et visqueuses sur le domaine de température $20-700{ }^{\circ} \mathrm{C}$ et l'on s'efforce d'identifier les mécanismes physiques importants contrôlant la déformation. 
Tableau I. - Composition pondérale de l'acier 17-12 Mo SPH en \%.

[Weight composition of the 17-12 Mo SPH steel in \%.]

\begin{tabular}{|c|c|c|c|c|c|c|c|c|c|c|c|}
\hline $\begin{array}{c}\mathrm{C} \\
\leqslant 0,03\end{array}$ & $\mathrm{~S}$ & $\mathrm{P}$ & $\mathrm{Si}$ & $\mathrm{Mn}$ & $\mathrm{Ni}$ & $\mathrm{Cr}$ & $\mathrm{Mo}$ & $\mathrm{N}$ & $\mathrm{B}$ & $\mathrm{Co}$ & $\mathrm{Cu}$ \\
& $\leqslant 0,001$ & $\leqslant 0,021$ & 0,44 & 1,084 & 12,3 & 17,54 & 2,47 & 0,075 & 0,001 & 0,15 & 0,175 \\
\hline $\begin{array}{c}\mathrm{Ti} \\
<0,005\end{array}$ & $\mathrm{Nb}$ & $\mathrm{Al}$ & $\mathrm{Ta}$ & $\mathrm{Fe}$ & $\mathrm{Fe}$ & 0,100 & - & $\mathrm{Bal}$. & &
\end{tabular}

\section{Méthodes expérimentales.}

Les éprouvettes sont obtenues à partir de lopins prélevés dans des tôles de $30 \mathrm{~mm}$ d'épaisseur, hypertrempées depuis $1200{ }^{\circ} \mathrm{C}$. La microstructure est entièrement austénitique et le diamètre moyen des grains est de $45 \mu \mathrm{m}$. La composition pondérale de cet acier élaboré par Creusot-Loire est donnée dans le tableau I.

Aucun traitement thermique n'est réalisé après l'usinage des différentes éprouvettes. Plusieurs machines ont été utilisées pour réaliser les essais, à savoir ; une machine hydraulique (traction), électrodynamique pilotées par microordinateur (tractiontorsion) et une machine de fluage à contraintes constantes (traction-torsion) [15]. Les éprouvettes de traction ont une longueur utile de $25 \mathrm{~mm}$ pour un diamètre de $5 \mathrm{~mm}$. Celles sollicitées en tractiontorsion ont une géométrie tubulaire dont les rayons extérieur et intérieur sont respectivement de 5 et $4 \mathrm{~mm}$ pour une longueur utile de $50 \mathrm{~mm}$.

\section{Résultats expérimentaux et discussion.}

\subsection{PRÉSENTATION GÉNÉRALE DES PROPRIÉtÉS MÉCANIQUES.}

3.1.1 Les essais monotones à vitesse imposée. - La figure 1 présente l'évolution de la contrainte d'écoulement $\tilde{\sigma}_{\text {ec }}$ pour une vitesse de déformation fixée, $\dot{\varepsilon}_{z z}^{\mathrm{T}}=6,6 \times 10^{-4} \mathrm{~s}^{-1}$ et pour différents niveaux de déformation $\tilde{\varepsilon}$, en fonction de la température ( $\tilde{\sigma}$ et $\tilde{\varepsilon}$ sont les contraintes et les déformations vraies). Il apparaît un plateau assez peu marqué entre 300 et $500{ }^{\circ} \mathrm{C}$. Pour les plus hautes températures $\left(T \geqslant 600{ }^{\circ} \mathrm{C}\right), \quad \tilde{\sigma}_{\text {ec }}$ décroît fortement, ce qui correspond à l'apparition des mécanismes de déformation thermiquement activés, pouvant conduire à l'écoulement stationnaire (à partir de $800^{\circ} \mathrm{C}$ pour cette vitesse de déformation).

Pour $T=525^{\circ} \mathrm{C}$, on reporte sur la figure 2 l'effet de la vitesse de sollicitation sur la contrainte d'écou-

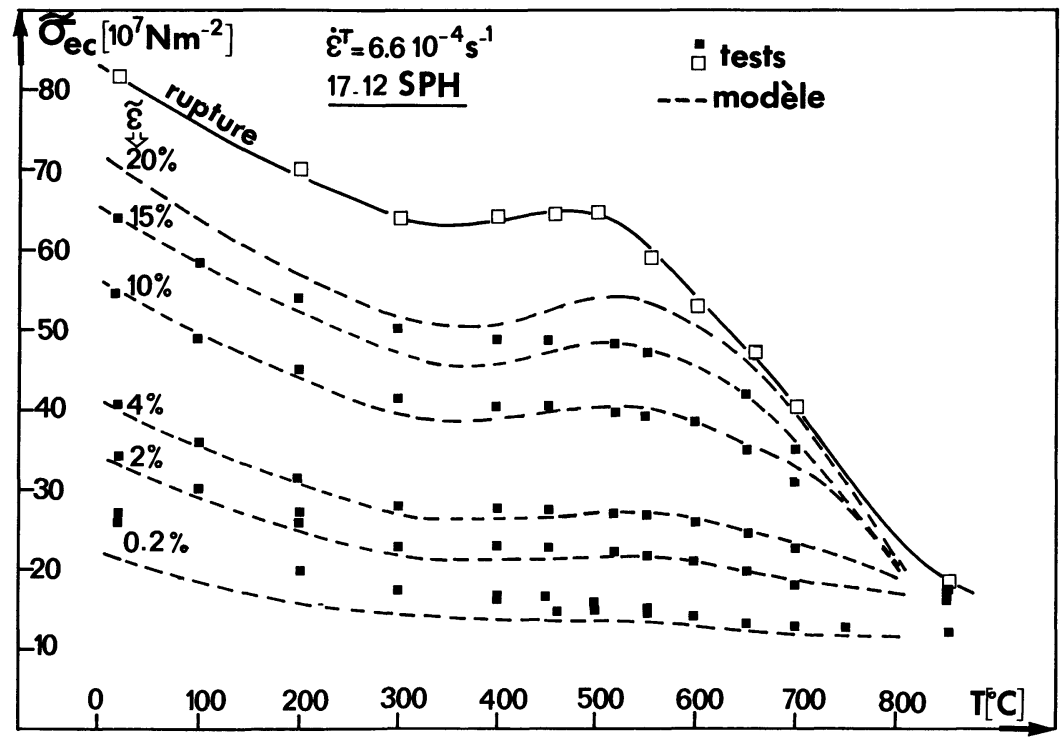

Fig. 1. - Evolution de la contrainte d'écoulement $\tilde{\sigma}_{\mathrm{ec}}$, pour différents niveaux de déformation, en fonction de la température. Expériences et modélisation.

[Evolution of the flow stress $\tilde{\sigma}_{\mathrm{ec}}$ for different levels of strain as a function of the temperature. Experiments and modelization.] 


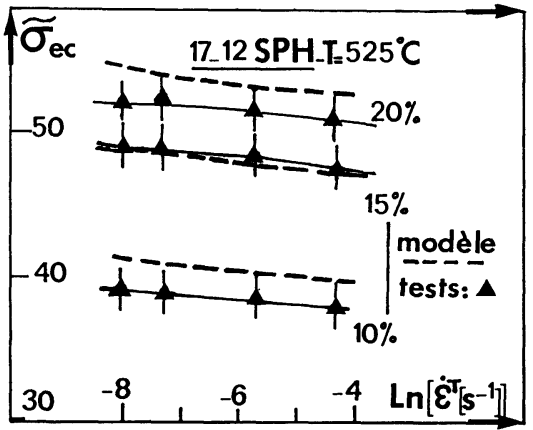

Fig. 2. - Effet de la vitesse sur la contrainte d'écoulement pour l'isotherme $T=525^{\circ} \mathrm{C}$ et pour trois niveaux de déformation. Essais et simulations.

[Effect of strain rate on the flow stress for the isotherm $T=525{ }^{\circ} \mathrm{C}$ and for three levels of strain. Tests and simulations.]

lement. Compte tenu des incertitudes estimées à partir de l'ampleur des hachures imputables au phénomène Portevin-Le Chatelier, on observe une quasi-insensibilité de la contrainte à la vitesse. Cependant, si l'on joint les points moyens, on révèle une tendance à la décroissance, le coefficient de sensibilité de la contrainte à la vitesse de sollicitation $\left(n=\left(\partial \tilde{\sigma}_{\mathrm{ec}} / \partial \operatorname{Ln} \dot{\varepsilon}_{\mathrm{zz}}^{\mathrm{T}}\right)_{\mathrm{T}}\right)$ pouvant être négatif. Cette tendance sera confirmée lors de l'étude des essais cycliques. On notera que les différents points qui viennent d'être évoqués (Figs. 1 et 2) corroborent les résultats de Matteazzi et al. [10].

\subsubsection{Les essais cycliques.}

3.1.2.1 Sollicitation unidirectionnelle (1D). - De nombreux essais de torsion cyclique ont été réalisés. On étudie, pour différentes isothermes $\left(20 \leqslant T \leqslant 650{ }^{\circ} \mathrm{C}\right)$, l'influence de l'amplitude de la déformation imposée $\left(\Delta \varepsilon_{z \theta}^{\mathrm{T}} / 2\right)$ et de la vitesse du cyclage $\dot{\varepsilon}_{z \theta}^{\mathrm{T}}$, sur le cycle stabilisé $\left(\Delta \sigma_{z \theta}^{\text {stab }} / 2\right)$. Ainsi, la figure 3 visualise, pour $\dot{\varepsilon}_{z \theta}^{\mathrm{T}}=4,6 \times 10^{-5} \mathrm{~s}^{-1}$, l'évolution de la contrainte stabilisée $\left(\Delta \sigma_{z \theta}^{\text {stab }} / 2\right)$, pour quatre amplitudes de déformation fixées (essais à niveaux successivement croissants), avec la température. On reporte également, pour le premier niveau $\left(\Delta \varepsilon_{z \theta}^{\mathrm{T}} / 2=3,5 \times 10^{-3}\right)$, le nombre de cycle $N_{\mathrm{c}}$ pour atteindre l'état stabilisé. Le comportement est très différent de celui observé sur la figure 1 et l'on montre qu'il se développe un pic très important dont le maximum se situe vers $550{ }^{\circ} \mathrm{C}$ et qui s'étale entre 200 et $600^{\circ} \mathrm{C}$, c'est-à-dire sensiblement la zone correspondant au plateau reporté sur les figures précédentes. On note qu'un tel maximum a déjà été observé sur un acier inoxydable du type 304 [16]. Ce maximum correspond également à un maximum de consolidation cyclique, l'écart entre le premier quart de cycle et le cycle stabilisé étant maximum (voir Fig. 1 par exemple).

La figure 4 donne par exemple, pour $T=600{ }^{\circ} \mathrm{C}$, une courbe de consolidation cyclique $(\Delta \bar{\sigma} / 2)=$ $f\left(\Delta \bar{\varepsilon}^{\mathrm{pl}} / 2\right)$, pour les sollicitations unidirectionnelles de traction et de torsion. $\left(\Delta \bar{\sigma}\right.$ et $\Delta \bar{\varepsilon}^{\mathrm{pl}}$, sont des équivalents au sens de Von-Misès décrits dans la nomenclature).

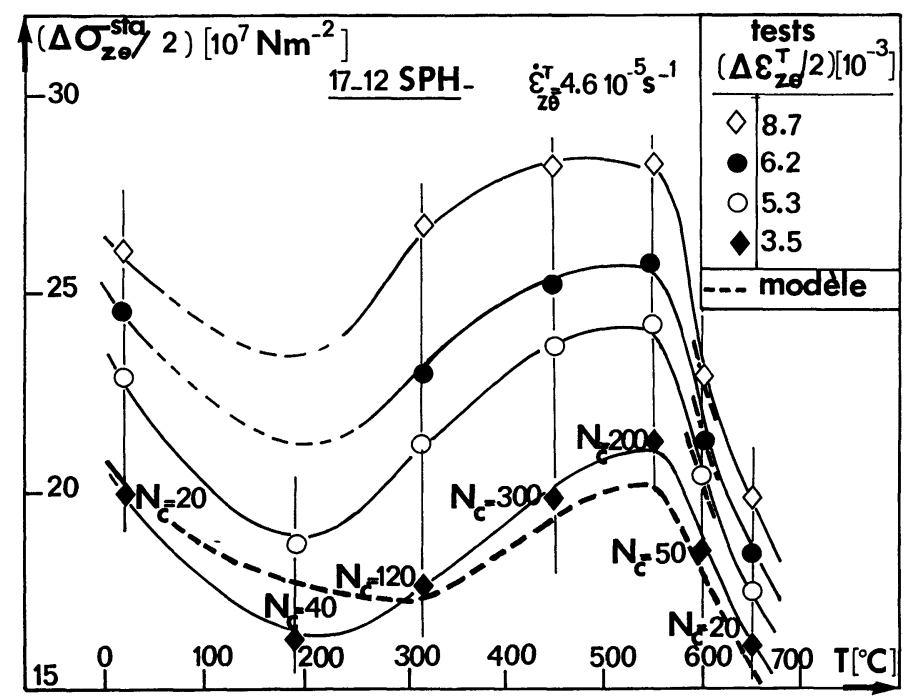

Fig. 3. - Evolution de la contrainte stabilisée $\left(\Delta \sigma_{z \theta}^{\text {stab }} / 2\right)$ en fonction de la température pour quatre niveaux de déformation $\left(\Delta \varepsilon_{z \theta}^{\mathrm{T}} / 2\right)$. Expériences et modélisation.

[Evolution of the stabilized stress $\left(\Delta \sigma_{z \theta}^{\text {stab }} / 2\right)$ as a function of the temperature for four levels of $\operatorname{strain}\left(\Delta \varepsilon_{z \theta}^{\mathrm{T}} / 2\right)$. Experiments and simulation.] 


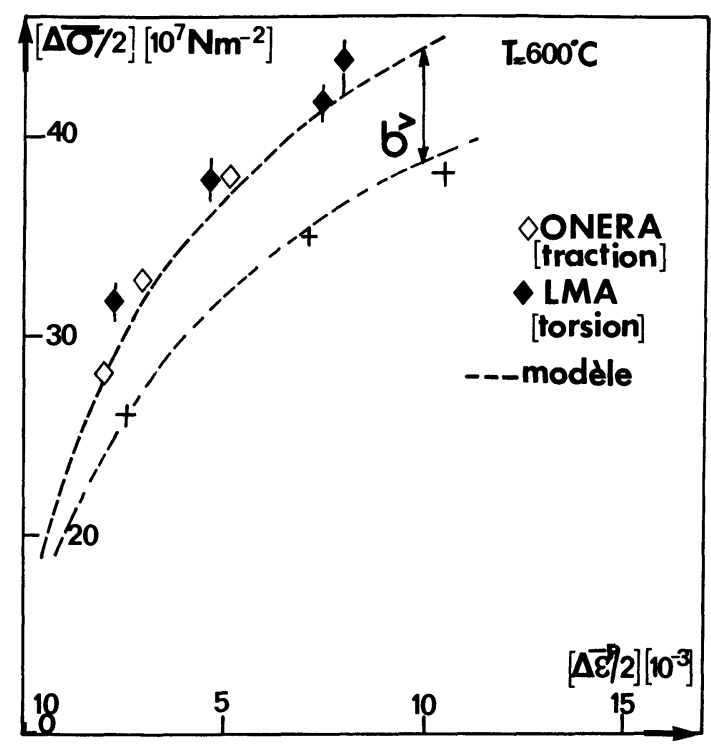

Fig. 4. - Courbe de consolidation cyclique à $600{ }^{\circ} \mathrm{C}$ et détermination expérimentale de la composante visqueuse $\widetilde{\sigma}_{\mathrm{v}}(+)$. Essais et modélisation.

[Cyclic hardening curve at $600{ }^{\circ} \mathrm{C}$. Experimental determination of the viscous component $\tilde{\sigma}_{v}(+)$. Tests and modelization.]

Si l'on s'intéresse aux effets de vitesse de déformation, dans un rapport de $10^{3}\left(1,38 \times 10^{-3} \leqslant \dot{\varepsilon}_{z \theta}^{\mathrm{T}} \leqslant\right.$ $1,38 \times 10^{-6} \mathrm{~s}^{-1}$ ), on montre figure 5 que le coefficient de sensibilité de la contrainte stabilisée à la vitesse $\left(n=\left(\frac{\partial\left(\Delta \sigma_{z \theta}^{\text {stab }} / 2\right)}{\partial \operatorname{Ln} \dot{\varepsilon}_{z \theta}^{\mathrm{T}}}\right){ }_{\mathrm{T}}\right)$ peut être positif ou négatif selon l'isotherme considérée. La valeur négative est observée dans le domaine des températures intermédiaires $\left(250 \leqslant T \leqslant 600{ }^{\circ} \mathrm{C}\right)$ et correspond exactement à celui du pic mentionné précédem-

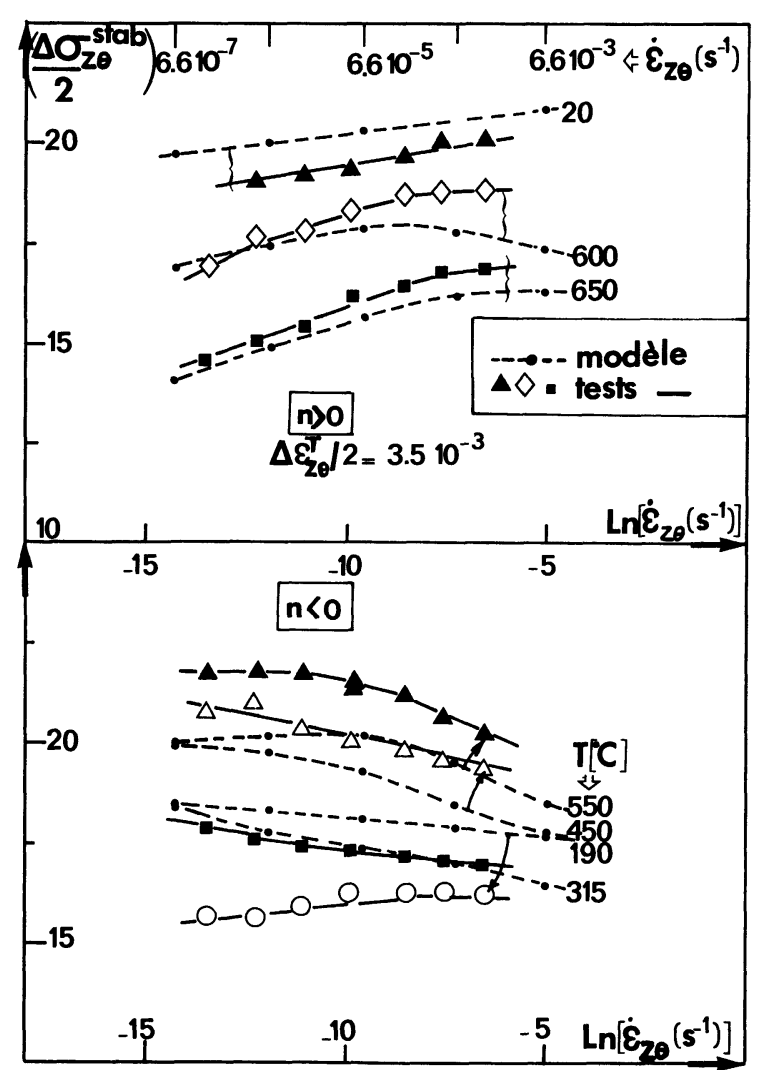

Fig. 5. - Effet de la vitesse $\dot{\varepsilon}_{z \theta}^{\mathrm{T}}$ sur le cycle stabilisé $\left(\Delta \sigma_{z \theta}^{\text {stab }} / 2\right)$ pour différentes isothermes. Essais et modélisation.

[Effect of the strain rate $\dot{\varepsilon}_{z \theta}^{\mathrm{T}}$ on the stabilized cycle $\left(\Delta \sigma_{z \theta}^{\text {stab }} / 2\right)$ for different isotherms. Tests and modelization.]

ment. On confirme ainsi la tendance issue de l'étude des essais monotones (Fig. 2). A basse température, ce coefficient est positif, ce qui traduit un effet de

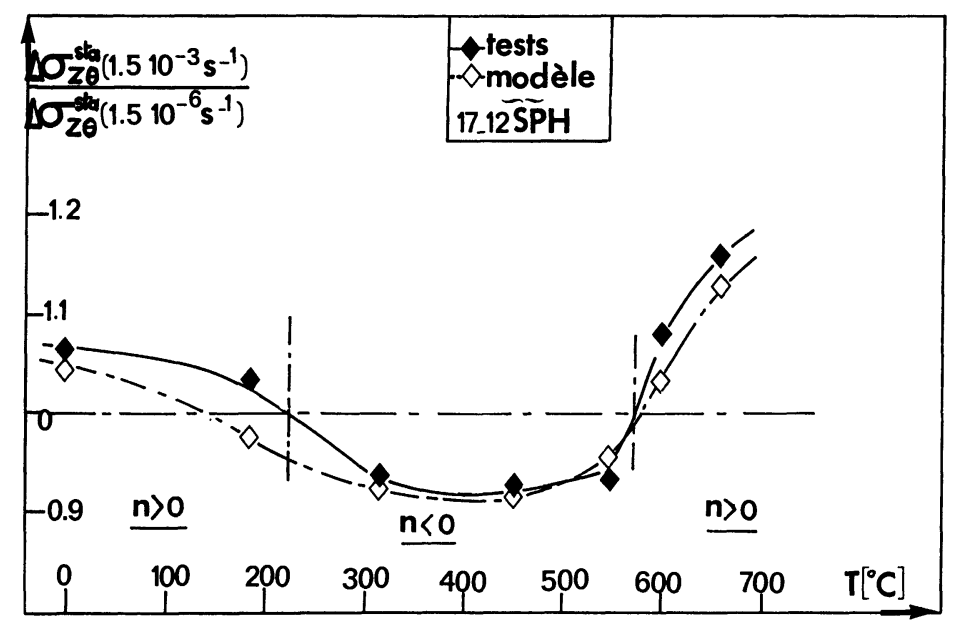

Fig. 6. - Evolution des rapports des contraintes stabilisées pour deux vitesses fixées dans un rapport de $10^{3}$. Expériences et modélisation.

[Evolution of the stabilized stress ratios for two fixed strain rates in a ratio of $10^{3}$. Experiments and modelization.] 
viscosité à froid (voir paragraphe ci-dessous). Pour les plus hautes températures $\left(T \geqslant 600^{\circ} \mathrm{C}\right)$, la sensibilité est naturellement positive puisque les effets de restauration thermiquement activée conduisent à une augmentation de la viscosité avec la température.

Pour résumer, on quantifie ces effets de vitesse, en s'affranchissant du niveau des contraintes atteintes, en calculant l'évolution du rapport des contraintes stabilisées pour un rapport de vitesse de $10^{3}$ (Fig. 6). Ce rapport peut être positif ou négatif.

3.1.2.2 Sollicitations 2D ; cas du rochet de tractiontorsion. - Sans entrer dans l'étude détaillée du phénomène de rochet $2 \mathrm{D}$ que l'on trouvera dans les références [17-12], on reporte quelques résultats significatifs corroborant ceux de la présente étude et conduisant à des constatations décisives pour la modélisation. On rappelle que la séquence expérimentale consiste en l'application d'une faible contrainte primaire de traction $\left(\sigma_{z z}\right)$ et en la superposition d'une déformation secondaire cyclique d'amplitude maximale constante $\left(\Delta \varepsilon_{z \theta}^{\mathrm{T}} / 2\right)$, pour une vitesse fixée $\left(\dot{\varepsilon}_{z \theta}^{\mathrm{T}}\right)$ et une isotherme donnée. La réponse de cet acier à ce type de sollicitation consiste en l'apparition d'une déformation progressive $\varepsilon_{z z}$, liée aux contraintes axiale $\sigma_{z z}$ et de cisaillement $\sigma_{z \theta}$, et en une consolidation cyclique suivant la composante de cisaillement. On peut donc étudier l'effet de la contrainte axiale $\sigma_{z z}$, de l'amplitude de la déformation de cisaillement, de la vitesse de cyclage et de la température sur la vitesse de déformation axiale cumulée $\dot{\varepsilon}_{z z}$ (effet de rochet) et sur l'amplitude du cycle stabilisé de torsion $\left(\Delta \sigma_{z \theta}^{\mathrm{stab}} / 2\right)[19]$.
Les conditions expérimentales des tests reportés correspondent à $\sigma_{z z}=50 \mathrm{MPa}, \Delta \varepsilon_{z \theta}^{\mathrm{T}} / 2= \pm 0,35 \%$, $\dot{\varepsilon}_{z \theta}^{\mathrm{T}}=4,6 \times 10^{-5} \mathrm{~s}^{-1}$ (période de $300 \mathrm{~s}$ ), pour différentes isothermes comprises entre 20 et $650{ }^{\circ} \mathrm{C}$. Les observations les plus significatives se résument de la manière suivante :

- la quasi-insensibilité du cycle de torsion au chargement primaire $\left(\Delta \sigma_{z \theta} / 2 \gg \sigma_{z z}\right)$, implique que l'évolution de la contrainte stabilisée $\left(\Delta \sigma_{z \theta}^{\text {stab }} / 2\right)$ en fonction de la température et de l'amplitude de cisaillement est tout à fait conforme aux résultats reportés sur la figure 3 ;

- de même, les effets de vitesse $\dot{\varepsilon}_{z \theta}^{\mathrm{T}}$ sur le cycle stabilisé sont identiques à ceux mentionnés sur la figure 5 ;

- par contre, la figure 7 représente, pour un nombre de cycles $N_{\mathrm{c}}$ fixé, l'évolution de la vitesse de déformation axiale cumulée $\dot{\varepsilon}_{z z}$ avec la température. Pour $N_{\mathrm{c}}$ suffisamment grand $\left(N_{\mathrm{c}}>200\right)$, il apparaît un minimum bien marqué vers $300{ }^{\circ} \mathrm{C}$, identique à celui observé sur la composante visqueuse (alinéa suivant). Pour $N_{\mathrm{c}}$ faible $\left(N_{\mathrm{c}}<100\right), \dot{\varepsilon}_{z z}$ croît d'une façon monotone avec la température. De plus, si l'on écrouit à froid le matériau, on montre (Fig. 7) que la vitesse de rochet pour $N_{\mathrm{c}}$ faible est affectée par ce préécrouissage, mais que, pour $N_{\mathrm{c}}$ grand $\left(N_{\mathrm{c}} \geqslant 500\right)$, celui-ci n'a aucun effet sur la vitesse. Ceci tend à montrer que l'on a sensiblement deux régimes d'écoulement que l'on pourrait qualifier de rochet «plastique » pour $N_{\mathrm{c}}$ faible et correspondant à l'établissement du cycle stabilisé, et de rochet « visqueux », pour $N_{\mathrm{c}}$ grand, correspondant sensiblement au fluage du matériau;

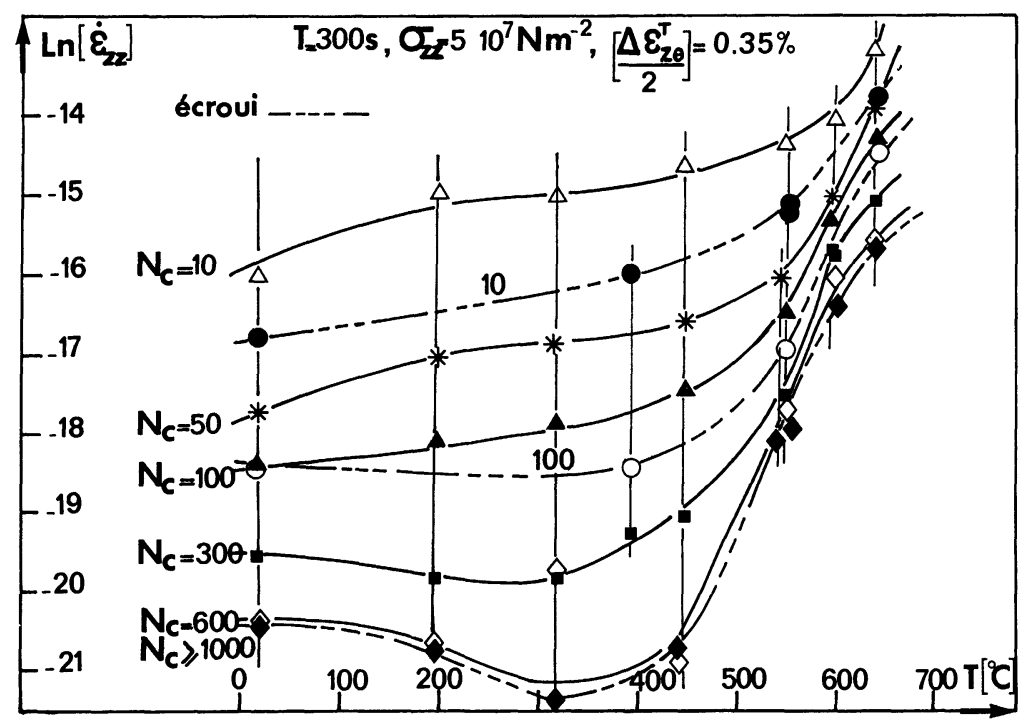

Fig. 7. - Evolution de la vitesse du rochet axial (rochet 2D) pour différentes isothermes et différents nombres de cycle $N_{\mathrm{c}}$. Influence de l'écrouissage.

[Evolution of the strain rate of axial ratchet (2D ratchet) for different isotherms and different numbers of cycles $N_{\mathrm{c}}$. Effect for the pre-hardening.] 
- l'analyse des trajectoires $\varepsilon_{z z}=f\left(\varepsilon_{z \theta}\right)$ consécutives à des perturbations réalisées sur le chargement primaire (décrément partiel ou total de $\sigma_{z z}$ ), pouvant conduire à du rochet négatif, est tout à fait conforme à la règle de normalité de la vitesse de déformation $\left(\dot{\varepsilon}_{z \theta}, \dot{\varepsilon}_{z z}\right)$ à des équipotentielles translatées cinématiquement [20-21]. La rotation continue de la direction d'écoulement, après l'inversion du sens de la vitesse de cisaillement jusqu'à l'inversion suivante, plaide également en faveur d'un comportement purement cinématique.

3.1.3 Estimation des propriétés visqueuses. - L'estimation de la composante visqueuse $\tilde{\sigma}_{\mathrm{v}}$ a été réalisée en utilisant la méthode de la relaxation inverse durant un essai à vitesse imposée [22] $\left(\dot{\varepsilon}_{z z}^{\mathrm{T}}=6,6 \times 10^{-4} \mathrm{~s}^{-1}\right)$. On rappelle que la composante visqueuse est définie par $\tilde{\sigma}_{\mathrm{v}}=\tilde{\sigma}-\tilde{\alpha}$ où $\tilde{\sigma}$ est la contrainte vraie appliquée et $\tilde{\alpha}$ est la somme des contributions des différentes contraintes internes à courte et longue portées. $\tilde{\alpha}$ correspond à l'obtention d'une vitesse de relaxation nulle consécutivement à un petit décrément de déformation.

On notera que ce type d'essai assez délicat a été réalisé sur une machine électrodynamique INSTRON 6025 pilotée par microordinateur et directement asservie sur la déformation de l'échantillon. Ces mesures ont été conduites pour différentes isothermes et pour des niveaux croissants de déformation, pratiquement jusqu'à rupture des éprouvettes. Les résultats sont reportés sur la figure 8 et conduisent à plusieurs commentaires :

- classiquement, $\tilde{\sigma}_{v}$ est une fonction croissante de la déformation qui peut atteindre une valeur stabilisée si l'écoulement stationnaire en contrainte est lui-même atteint $[7,23]$;
- la composante visqueuse présente un minimum entre 250 et $400{ }^{\circ} \mathrm{C}$. Ce constat confirme les observations réalisées sur la figure 7 , quant à l'évolution de la vitesse de rochet axial $\dot{\varepsilon}_{z z}$ avec la température, pour $N_{\mathrm{c}}$ grand. On montre également que cet acier présente une viscosité assez conséquente au voisinage de la température ambiante. Pour les hautes températures, la croissance de $\tilde{\sigma}_{\mathrm{v}}$ est liée à l'apparition des phénomènes thermiquement activés;

- on remarquera qu'au-delà de $650^{\circ} \mathrm{C}$ et pour les plus forts niveaux de déformation, les différentes hypothèses sous-jacentes à cette technique (figeage de la microstructure pendant quelques secondes), risquent, compte tenu de la rapidité de la restauration de la microstructure, de n'être plus respectées. Les résultats de ces mesures sont donc à regarder avec circonspection.

La technique expérimentale du retour de limite d'élasticité présentée dans le paragraphe suivant, permet d'accéder (Fig. 10), pour différentes isothermes et différents niveaux de déformation, à l'amplitude relaxée $\widetilde{\Delta \sigma}_{\text {rel }}$ au bout d'un temps fixé $t_{\mathrm{v}}$. On reporte, figure 9 , l'évolution de cette amplitude avec la température, pour $t_{\mathrm{v}}=48 \mathrm{~h}$. On confirme, à nouveau, les résultats issus de l'estimation de la composante visqueuse par la méthode de la relaxation inverse, à savoir, la perte de viscosité entre 200 et $400^{\circ} \mathrm{C}$. En effet, en l'absence de phénomènes dépendant du temps $\left(T \leqslant 600{ }^{\circ} \mathrm{C}\right)$, l'amplitude maximale relaxée $\left(t_{\mathrm{v}} \rightarrow \infty\right)$ doit tendre vers $\tilde{\sigma}_{\mathrm{v}}$.

En conclusion, on peut affirmer que la perte de viscosité sur le domaine des températures intermédiaires correspond sensiblement à la zone d'apparition des effets de sensibilité négative à la vitesse, au plateau sur les contraintes d'écoulement sous sollici-

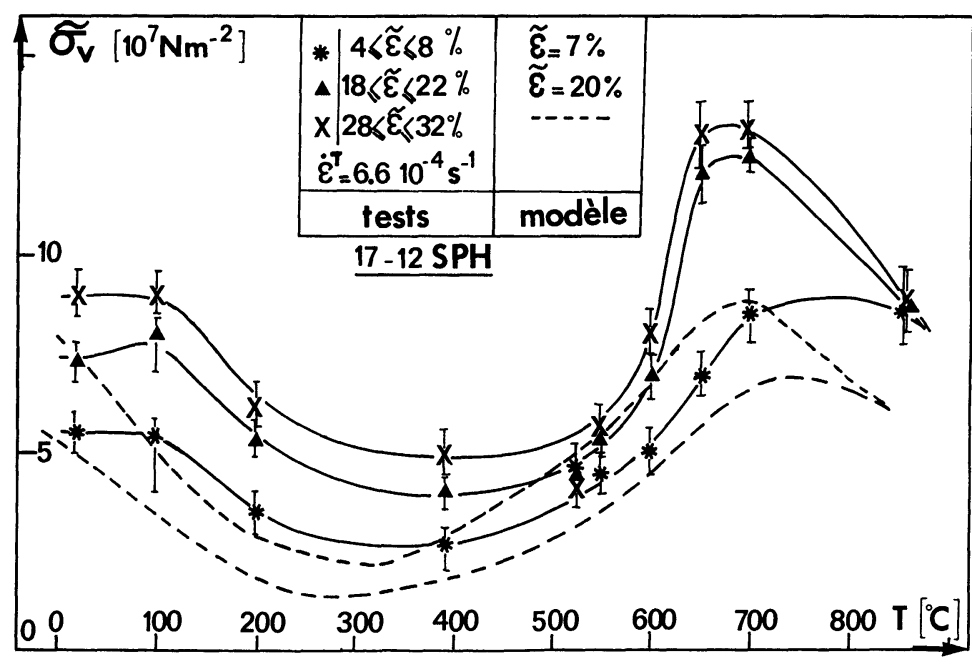

Fig. 8. - Evolution de la contrainte visqueuse $\tilde{\sigma}_{\mathrm{v}}$ avec la température, pour trois niveaux de déformation. Expériences et modélisation.

[Evolution of the viscous stress $\tilde{\sigma}_{\mathrm{v}}$ with the temperature for three levels of strain. Experiments and modelization.] 


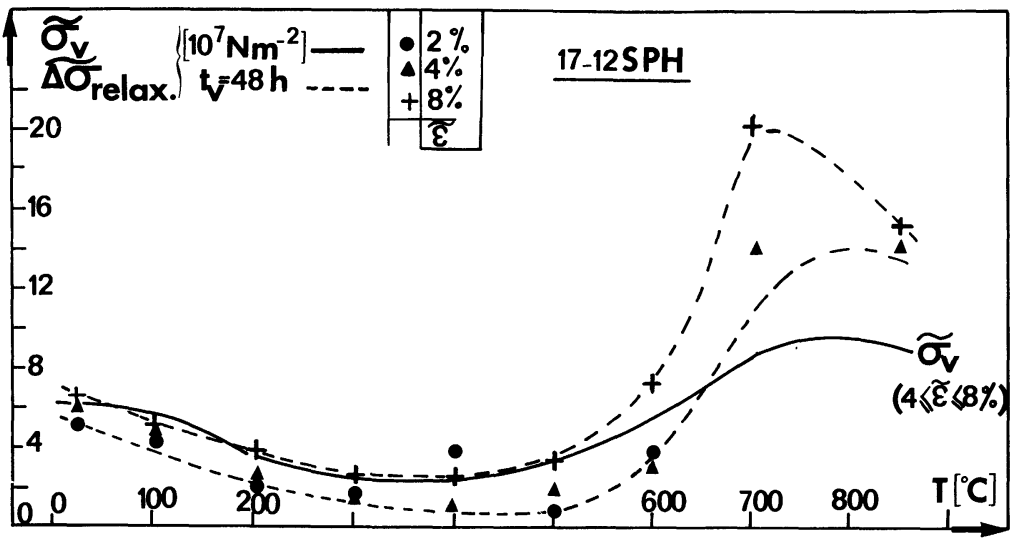

Fig. 9. - Evolution de l'amplitude relaxée, $\widetilde{\Delta \sigma}_{\text {rel }}$, en $48 \mathrm{~h}$ pour différents niveaux de déformation. Comparaison avec l'évolution de la composante visqueuse $\tilde{\sigma}_{\mathrm{v}}$.

[Evolution of the relaxed amplitude, $\widetilde{\Delta \sigma}_{\text {rel }}$, in 48 hours for different levels of strain. Comparison with the evolution of the viscous component $\tilde{\sigma}_{\mathrm{v}}$.]

tation monotone et au maximum sur la contrainte stabilisée sous sollicitation cyclique. Une présentation synthétique des propriétés thermomécaniques de cet acier est fournie dans le tableau II.

\subsection{MÉCANISMES DE DÉFORMATION AUX TEMPÉRA-} TURES INTERMÉDIAIRES. - De façon à corréler à un paramètre physique les différents phénomènes cités précédemment, nous avons réalisé des mesures d'évolution de seuil d'écoulement, conformément au schéma de principe de la figure 10 . On quantifie les variations de limite d'élasticité par le paramètre $\widetilde{\Delta \sigma}_{\mathrm{r}}=\tilde{\sigma}_{\mathrm{e}}-\tilde{\sigma}(0)$ et l'on étudie l'influence de la température, de la déformation et du temps de la relaxation $t_{\mathrm{v}}$, la vitesse de traction imposée ayant été fixée à $6,6 \times 10^{-4} \mathrm{~s}^{-1}$. Conformément au schéma de la figure 10 , on constate que suivant la température étudiée, $\widetilde{\Delta \sigma}_{\mathrm{r}}$ peut être positif pour les plus basses températures ou négatif pour les plus élevées. On illustre notre propos en reportant, figure 11, quelques enregistrements correspondant à $\widetilde{\Delta \sigma}_{\mathrm{r}}>0$, pour $T=200$ et $300{ }^{\circ} \mathrm{C}$.

Quantitativement, on reporte sur la figure 12 l'évolution de $\widetilde{\Delta \sigma}_{\mathrm{r}}$ en fonction de la température, pour $t_{\mathrm{v}}=48 \mathrm{~h}$ et différents niveaux de déformation. Pour $T \geqslant 550{ }^{\circ} \mathrm{C}, \widetilde{\Delta \sigma}_{\mathrm{r}}$ est négatif, ce qui est imputable à la restauration par le temps et qui conduit au fluage stationnaire thermiquement activé [7, 24], pour $T<550{ }^{\circ} \mathrm{C}, \widetilde{\Delta \sigma_{\mathrm{r}}}$ est positif, ce qui résulte d'un durcissement par le temps mais évanescent avec la déformation (Fig. 11). On notera que, dans la zone de transition $\widetilde{\Delta \sigma}_{\mathrm{r}} \gtrless 0$, il apparaît les phénomènes d'instabilité du type Portevin-Le Chatelier sur les courbes effort-déformation. La figure 13 montre que

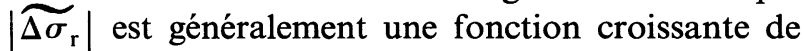
la déformation, excepté pour les températures de transition (vers $550{ }^{\circ} \mathrm{C}$ ) où les phénomènes de durcissement et d'adoucissement par le temps coexistent et

Tableau II. - Résumé des propriétés mécaniques de l'acier 17-12 SPH.

[Summary of the mechanical properties of the 17-12 SPH steel.]

\begin{tabular}{|c|c|c|}
\hline - fluage à froid & $\begin{array}{l}\text { - pas ou très peu de déformation } \\
\text { dépendante du temps }\end{array}$ & $\begin{array}{l}\text { - fluage à haute température, } \\
\text { thermiquement activé }\end{array}$ \\
\hline - rochet $(1 \mathrm{D}$ et $2 \mathrm{D})$ & - peu de rochet (1D et $2 \mathrm{D})$ & $\begin{array}{l}\text { - rochet }(1 \mathrm{D} \text { et } 2 \mathrm{D}) \\
\text { thermiquement activé }\end{array}$ \\
\hline $\begin{array}{l}\text { - peu de consolidation } \\
\text { cyclique }\end{array}$ & $\begin{array}{l}\text { - très forte consolidation } \\
\text { cyclique }\end{array}$ & $\begin{array}{l}\text { - consolidation cyclique assez } \\
\text { importante avec effet du temps }\end{array}$ \\
\hline $\begin{array}{l}\text { - sensibilité positive de la } \\
\text { contrainte à la vitesse }\end{array}$ & $\begin{array}{l}\text { - sensibilité négative de la } \\
\text { contrainte à la vitesse }\end{array}$ & $\begin{array}{l}\text { - sensibilité positive de la } \\
\text { contrainte à la vitesse }\end{array}$ \\
\hline $20^{\circ} \leqslant T<200^{\circ} \mathrm{C}$ & $200<T<550^{\circ} \mathrm{C}$ & $T \geqslant 550^{\circ} \mathrm{C}$ \\
\hline
\end{tabular}




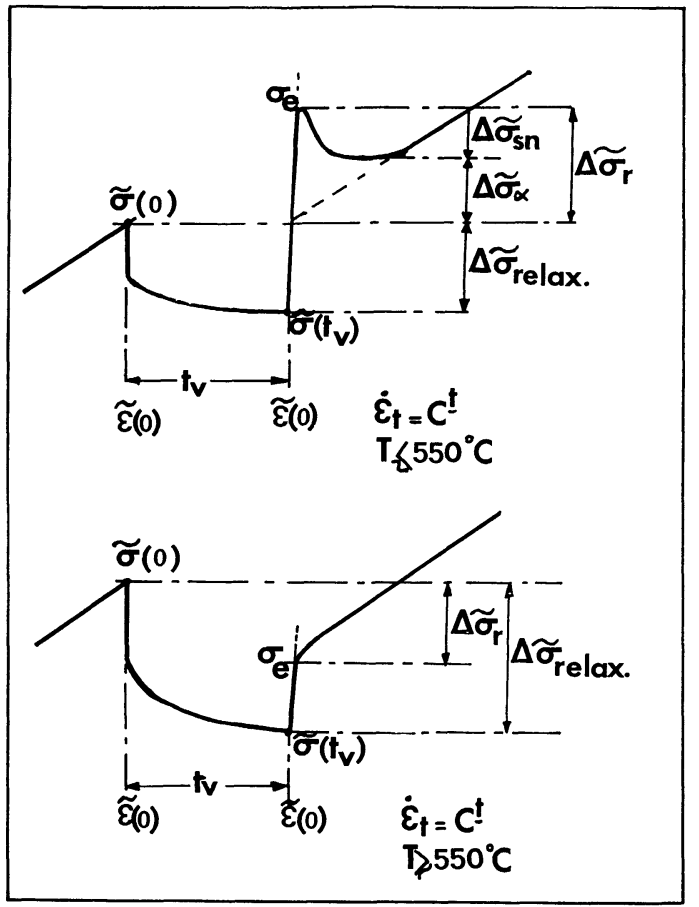

Fig. 10. - Schéma de principe de la méthode du retour de limite d'élasticité.

[Diagram of the principle of the method of yield point return.]

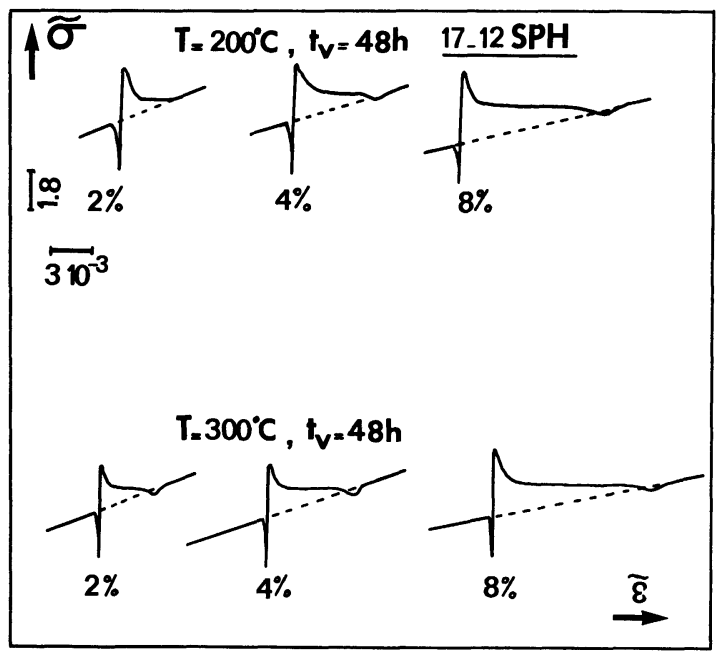

Fig. 11. - Exemples d'accroissement de limite d'élasticité, $\widetilde{\Delta \sigma}_{\mathrm{r}}>0$.

[Examples of the augmentation of the elastic limit, $\widetilde{\Delta \sigma}{ }_{\mathrm{r}}>0$.]

peuvent successivement, suivant le niveau de déformation, contrôler l'évolution de $\widetilde{\Delta \sigma}_{\mathrm{r}}$.

En conclusion, on montre que le domaine d'apparition des comportements "anormaux " décrits au paragraphe 3.1 correspond sensiblement à la plage où $\widetilde{\Delta \sigma}_{\mathrm{r}}$ est positif. En accord avec les travaux de

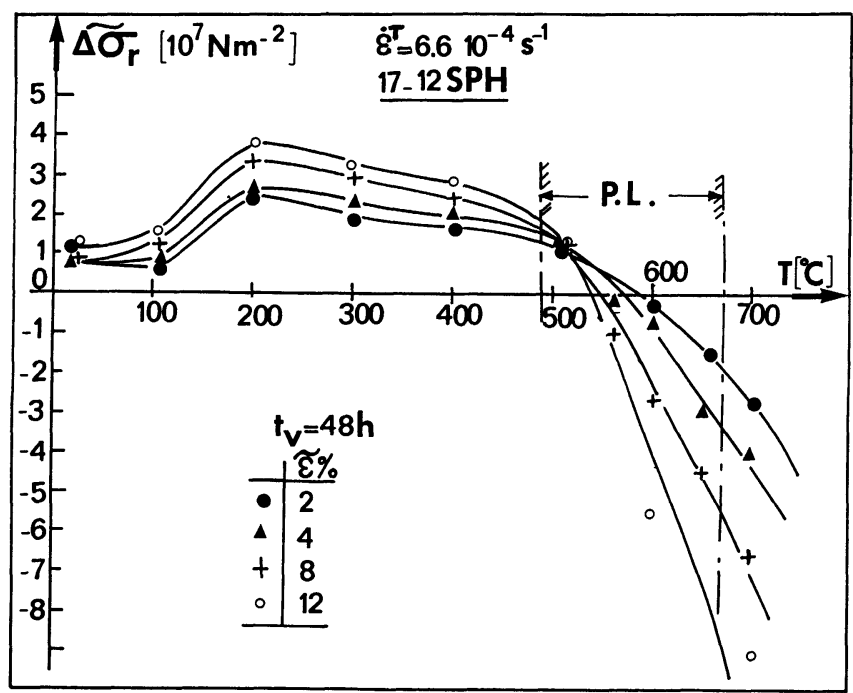

Fig. 12. - Evolution du paramètre $\widetilde{\Delta \sigma}_{\mathrm{r}}$ avec la température, pour $t_{\mathrm{v}}=48 \mathrm{~h}$ et quatre niveaux de déformation. Mise en évidence de $\widetilde{\Delta \sigma}_{\mathrm{r}}>0$ ou $\widetilde{\Delta \sigma}_{\mathrm{r}}<0$.

[Evolution of the parameter $\widetilde{\Delta \sigma}_{\mathrm{r}}$ with the temperature, for $t_{\mathrm{v}}=48 \mathrm{~h}$ and four levels of strain. Condition of $\widetilde{\Delta \sigma}_{\mathrm{r}}>0$ or $\widetilde{\Delta \sigma}_{\mathrm{r}}<0$ made evident.]

Blanc [13] conduits sur un acier très voisin, cette zone correspond au domaine d'interactions fortes entre dislocations et défauts ponctuels. On attribuera donc, aux interactions entre dislocations et défauts ponctuels, une composante importante du comportement de cet acier aux températures intermédiaires. D'un point de vue microscopique, on mentionne que l'unanimité n'est pas réalisée quant à l'origine du blocage des dislocations par les défauts ponctuels et l'on peut citer, d'une manière non exhaustive, les références [25-28].

L'étude quantitative de la cinétique de $\widetilde{\Delta \sigma_{\mathrm{r}}}$ $\left(\widetilde{\Delta \sigma}_{\mathrm{r}}=f\left(t_{\mathrm{v}}\right)\right)$ pour différentes isothermes, peut conduire à quelques informations physiques sur l'origine des mécanismes de blocage. Dans cette optique, on reporte, figure 14, l'évolution de la quantité normalisée

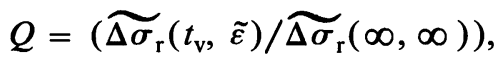

en fonction du temps $t_{\mathrm{v}}$ de vieillissement sous contrainte (temps de la relaxation). Pour chaque isotherme, $\widetilde{\Delta \sigma}_{\mathrm{r}}(\infty, \infty)$ correspond à la valeur asymptotique de $\widetilde{\Delta \sigma}_{\mathrm{r}}\left(t_{\mathrm{v}}, \tilde{\varepsilon}\right)$ lorsque $t_{\mathrm{v}}$ et $\tilde{\varepsilon}$ tendent vers l'infini, c'est-à-dire expérimentalement, lorsque l'on n'observe plus d'évolution notable de $\widetilde{\Delta \sigma}_{\mathrm{r}}$ lorsque $t_{\mathrm{v}}$ augmente et lorsque $\tilde{\varepsilon}=20 \%$ (Fig. 13). De cette façon, on s'affranchit du niveau de la déformation.

Pour chaque isotherme, l'évolution de $Q$ n'a pas une forme simple mais présente plusieurs oscillations caractéristiques de plusieurs cinétiques d'interaction 


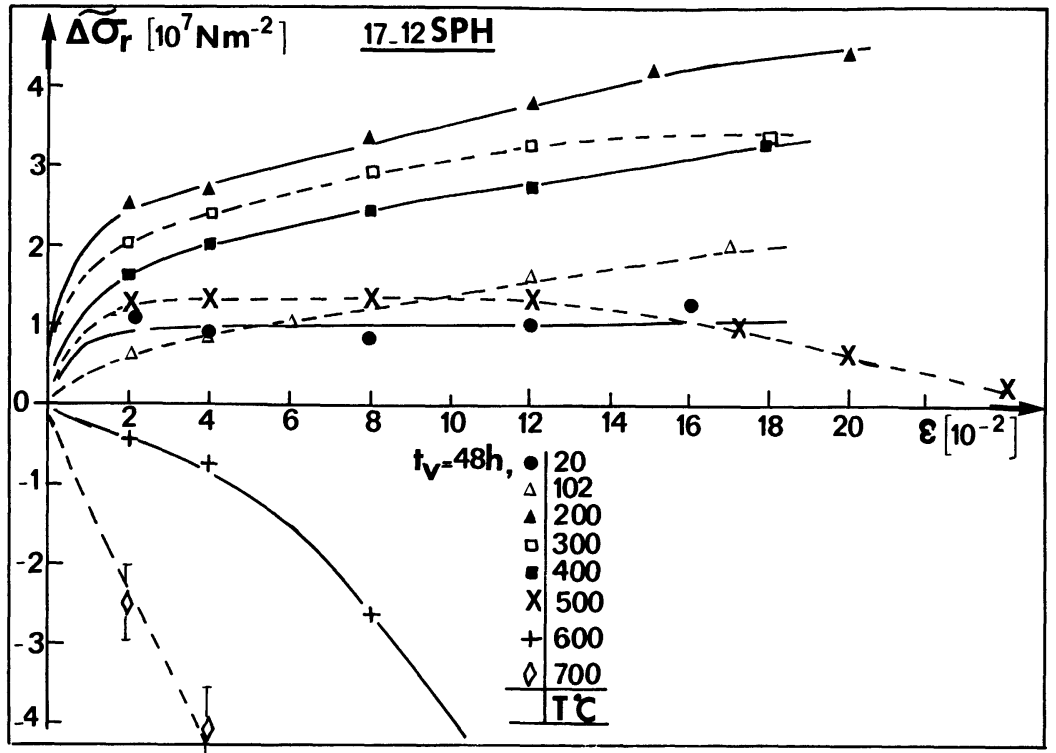

Fig. 13. - Evolution de $\widetilde{\Delta \sigma}$ avec la déformation, pour $t_{\mathrm{v}}=48 \mathrm{~h}$ et différentes isothermes.

[Evolution of $\widetilde{\Delta \sigma_{\mathrm{r}}}$ with the strain, for $t_{\mathrm{v}}=48 \mathrm{~h}$ and different isotherms.]

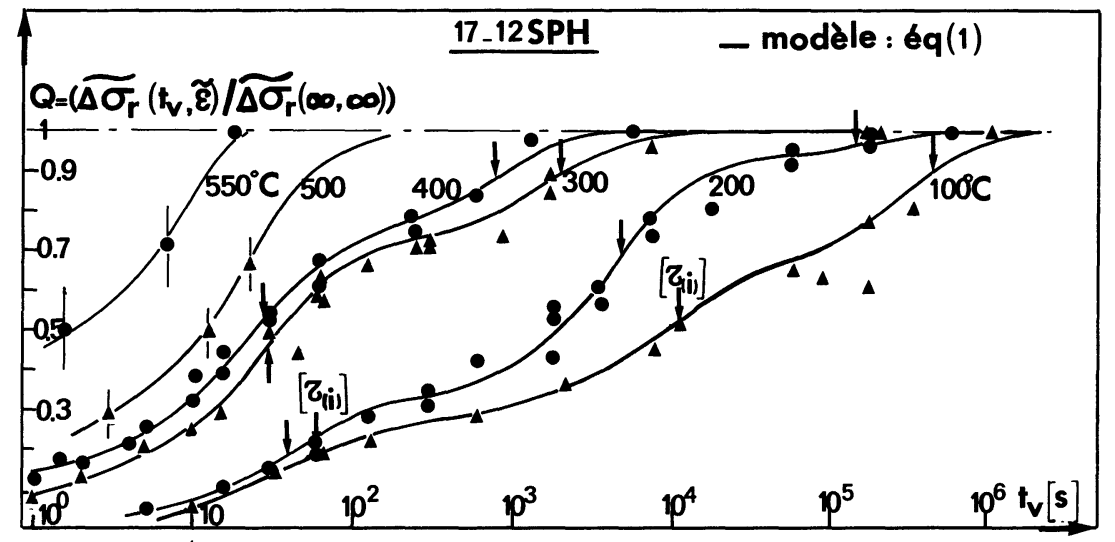

Fig. 14. - Etude de la cinétique de vieillissement pour différentes isothermes, $Q=\widetilde{\Delta \sigma}_{\mathrm{r}}\left(t_{\mathrm{v}}, \tilde{\varepsilon}\right) / \widetilde{\Delta \sigma_{\mathrm{r}}}(\infty, \infty)=f\left(t_{\mathrm{v}}\right)$. Expériences et modélisation.

[Study of the kinetics of aging for different isotherms, $Q=\widetilde{\Delta \sigma}_{\mathrm{r}}\left(t_{\mathrm{v}}, \tilde{\varepsilon}\right) / \widetilde{\Delta \sigma}_{\mathrm{r}}(\infty, \infty)=f\left(t_{\mathrm{v}}\right)$. Experiments and modelization.]

qui contribuent d'une manière additive au durcissement global du matériau [29-31]. Un tel comportement a déjà été observé pour des solutions solides C.C. plus simples, à savoir le Fe-C-N [30] et le Ta$\mathrm{O}-\mathrm{N}[31,47]$. En prenant des cinétiques du premier ordre, ce que nous justifierons ultérieurement, on écrit pour chaque isotherme :

$$
Q=\sum_{(i)} q_{(i)}\left[1-\exp -\frac{t_{\mathrm{v}}}{\tau^{(i)}}\right],
$$

(i) correspond au nombre d'oscillations sur une courbe $Q=f\left(t_{\mathrm{v}}\right)$, pour une isotherme donnée, $q_{(i)}$ est l'amplitude de l'oscillation (i) et $\tau^{(i)}$ sa constante de temps. Les courbes ajustées par cette technique sont tracées sur la figure 14. Le report des constantes de temps $\tau^{(i)}$ dans des coordonnées d'Arrhénius, $\operatorname{Ln} \tau^{(i)}=f(1 / T)$, figure 15, permet d'une part de dénombrer au moins cinq interactions numérotées de 1 à 5 sur la figure 15 , et d'autre part, de déterminer les énergies d'activation $W^{(i)}$ par la pente des droites $\operatorname{Ln} \tau^{(i)}=f(1 / T)$, conformément à l'équation (2) :

$$
\tau^{(i)}=\tau_{0}^{(i)} \exp \frac{W^{(i)}}{k T}
$$

On obtient $0,9( \pm 0,05) \leq W^{(i)} \leq 1,2( \pm 0,05) \mathrm{eV}$. 


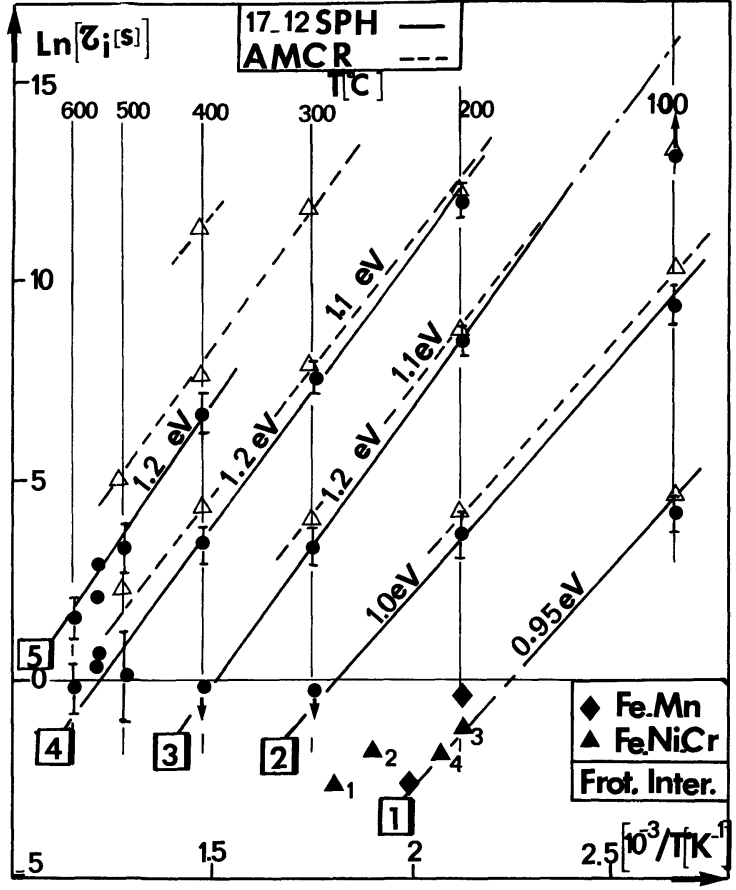

Fig. 15. - Diagramme d'Arrhénius, $\operatorname{Ln} \tau_{(i)}=f(1 / T)$, pour les deux aciers inoxydables 17-12 SPH et AMCR 033. Détermination des énergies d'activation $W_{(i)}$. (Eq. (2)).

[Arrhenius diagram, $\operatorname{Ln} \tau_{(i)}=f(1 / T)$, for two stainless steels : 17-12 SPH and AMCR 033. Determination of the activation energies $W_{(i)}$ (Eq. (2)).]

On signale que les points reportés à 550 et $600{ }^{\circ} \mathrm{C}$ correspondent directement aux temps de montée des hachures en dents de scie observées sur les courbes efforts-déformations pour différentes vitesses de sollicitation (phénomène Portevin-Le Chatelier). Les temps de relaxation déduits des pics de frottement interne observés au voisinage de $1 \mathrm{~Hz}$ sur des aciers $\mathrm{Fe}-\mathrm{Cr}-\mathrm{Ni}-\mathrm{N}$ [32] et $\mathrm{Fe}-\mathrm{Mn}-\mathrm{C}[33,34]$ sont également reproduits sur la figure 15 . On mentionne que pour l'acier au nickel-chrome, Dubois et al. [32] décompose le spectre d'amortissement en deux souspics d'où les quatre points notés sur la figure 15 . On reporte également, à titre de comparaison, les points obtenus, par la technique du retour de limite d'élasticité, sur un acier inoxydable austénitique $\mathrm{Fe}-\mathrm{Mn}-\mathrm{Cr}$ (AMCR) $[35,36]$ et l'on montre ainsi la proximité des temps de relaxation mesurés dans ces deux aciers.

A ce stade de l'étude, plusieurs commentaires s'imposent : pour ce qui concerne la détermination des temps de relaxation $\tau^{(i)}$, donc des énergies d'activation $W^{(i)}$, on mentionne d'une part l'incertitude liée à la technique expérimentale et à la séquence de normalisation de la courbe cinétique (détermination de $Q$ ) et d'aute part, la subjectivité quant au nombre d'oscillations observées pour chaque isotherme (Fig. 14). En effet, pour les températures étudiées comprises entre 100 et $400{ }^{\circ} \mathrm{C}$, on reporte trois oscillations, ce qui peut présenter un caractère subjectif, mais il semble clair qu'au minimum deux cinétiques soient nécessaires pour lisser les courbes expérimentales. Une seule cinétique du premier ordre s'avère être très insuffisante. La subjectivité et la non-unicité mathématique de la solution se retrouve dans l'étude de phénomènes voisins, liés à la présence de défauts ponctuels, par la technique du frottement interne où il est très fréquent de décomposer le spectre global d'amortissement en une somme de deux à cinq contributions élémentaires du type Debye [34]. Le fait qu'il n'apparaît que trois oscillations pour chaque isotherme (Fig. 14), alors que la figure 15 permet de déterminer cinq droites donc autant de mécanismes élémentaires, peut s'expliquer en introduisant, comme nous le ferons ultérieurement dans la modélisation, la fonction d'efficacité de chaque contribution au durcissement global du matériau. Ainsi, une configuration peut être potentiellement probable, donc éventuellement s'intégrer dans le diagramme d'Arrhénius de la figure 15, sans pour autant contribuer de manière décisive au durcissement global du matériau (Fig.14). On peut illustrer notre propos à l'aide de la figure 17 où l'on observe que pour une verticale correspondant à une isotherme donnée, le nombre d'intersections avec les différentes fonctions d'efficacité (repères 1 à 5) est toujours compris entre un et trois.

Enfin, notons que les incertitudes sur la détermination des paramètres $q^{(i)}$ (Eq. (1)) sont globalement pondérées dans l'évaluation quantitative du modèle par l'introduction d'une constante de répartition $\gamma_{\mathrm{c}}$ dans l'équation (18). Ces réserves étant posées, il est néanmoins possible de discuter les valeurs des énergies d'activation déterminées sur la figure 15 .

Si l'on adopte une autre démarche en s'affranchissant des oscillations obtenues expérimentalement, c'est-à-dire que l'on abandonne l'hypothèse des différentes réorientations possibles, et que l'on cherche à décrire l'allure générale des courbes à l'aide d'une seule cinétique d'ordre différent de un [40-42], soit :

$$
Q=q\left(1-\exp -\left(\frac{t_{\mathrm{v}}}{\tau}\right)^{n_{i}}\right) \text { avec } \tau=\tau_{0} \exp \frac{W}{k T},
$$

on peut trouver, moyennant un lissage grossier, $n_{i}=0,5$ et $W=1,0 \pm 0,1 \mathrm{eV}$. L'énergie d'activation ainsi calculée est voisine de celles déterminées par la méthode précédente, ce qui tend à renforcer la signification physique de cette valeur :

- bien qu'entachées d'imprécisions expérimentales, les valeurs des énergies d'activation calculées sont bornées par l'énergie de self-diffusion du carbone dans l'austénite $\left(W_{\mathrm{c}} \simeq 1,4 \mathrm{eV}\right)$ [37] et l'énergie de migration d'une lacune dans l'austénite $\left(W_{\ell} \simeq 0,9 \mathrm{eV}\right)[37,38]$. Les deux pics de frottement 
interne reportés par Dubois et al. [32] et liés à la présence d'azote, ont pour énergie 0,95 et $1,2 \mathrm{eV}$, ce qui corrobore nos déterminations. On peut donc supputer, en accord avec quelques auteurs [12, 13, 29-32, 38], que les atomes interstitiels (C, $\mathbf{N}$ et éventuellement B) sont responsables du blocage des dislocations ;

- la nature précise des mécanismes de blocage est plus incertaine, mais compte tenu, d'une part des basses températures où apparaissent ces phénomènes (absence de diffusion à longue distance) et d'autre part, de la proximité des temps de relaxation obtenus en frottement interne et ceux de la présente étude, nous penchons pour des phénomènes d'interactions à courte distance du type Snoek-Shoeck dans les solutions solides du type C.F.C. [27, 39]. Avec ce type de réseau, pour que le réarrangement des défauts ponctuels puisse se produire dans le champ des contraintes engendrées par les dislocations et donnant lieu, soit aux phénomènes décrits précédemment, soit à une relaxation anélastique, il faut que la symétrie des défauts soit inférieure à celle du réseau, c'est-à-dire qu'un défaut interstitiel isolé doit se combiner à un autre défaut voisin en position interstitielle (i), substitutionnelle (s) ou lacunaire $(\ell)$ pour constituer un dipôle ou une configuration de défauts ponctuels [32, 34, 38]. Notons que pour un réseau C.C., un seul atome interstitiel peut donner lieu à ce mécanisme de blocage que quelques auteurs ont identifié comme étant responsable du premier stade de vieillissement de quelques solutions solides : $\mathrm{Nb}-\mathrm{O}[29,31,40,41]$, Ta-O-N [30, 42], Fe-C-N [30, 43, 44], Va-O [45], Ni-C [46] ;

- si l'on admet l'existence d'un mécanisme du type Snoek, la cinétique de réorientation des dipôles dans le champ de contrainte des dislocations est du premier ordre $[27,29,30,34,47,48]$ et si $U$ est la profondeur de la vallée de potentiel dans laquelle se trouve une dislocation en configuration de blocage, on montre [27, 39] que :

$$
\frac{\mathrm{d} U}{\mathrm{~d} t}=\left(\frac{U_{\infty}-U}{\tau}\right)
$$

L'équation (3), sous une forme différentielle, justifie l'utilisation de la cinétique de l'équation (1). $U_{\infty}$ est liée à l'augmentation de la contrainte $\left(\widetilde{\Delta \sigma}_{\mathrm{r}(i)}\right)$ nécessaire pour extraire une dislocation de sa vallée de potentiel créée par la réorientation de la configuration $(i)[27,39]$;

- les différentes configurations (i) contribuent d'une manière additive au mécanisme de blocage $[34,30,31,47,49]$, ce qui justifie la sommation sur l'indice $(i)$ dans l'équation (1);

- bien évidemment, le nombre de configurations réorientées est lié à la densité des dislocations mobiles, ce qui justifie l'augmentation de $\widetilde{\Delta \sigma_{\mathrm{r}}}$ avec la déformation (Fig. 13) [29, 31, 46-48] ;
- la nature exacte des atomes des configurations susceptibles de se réorienter est très difficile à déterminer compte tenu de la complexité de la composition de cet acier. Néanmoins, le carbone et l'azote semblent intervenir de façon impérative, le nickel, le manganèse et le chrome en position substitutionnelle, peuvent éventuellement former des dipôles avec le carbone et l'azote. Ceci étaye les conclusions de Dubois et. al. [32] qui attribuent la partie basse température du spectre de frottement interne à la présence de paires N-N $\left(W_{\mathrm{N}-\mathrm{N}}=0,96 \mathrm{eV}\right)$ et la partie haute température aux paires $\mathrm{N}-\mathrm{Cr}\left(W_{\mathrm{N}-\mathrm{Cr}}=1,2 \mathrm{eV}\right)$. Le dipôle C-C semble avoir une énergie trop élevée $\left(W_{\mathrm{C}-\mathrm{C}}=1,43 \mathrm{eV}\right)$ par rapport à celles calculées, par contre, l'association C-Lacune $\left(W_{\mathrm{C}-\mathrm{L}}=0,87 \mathrm{eV}\right)$ est une configuration tout à fait probable [38].

\section{Vers une nouvelle formulation anisotherme d'un modèle viscoplastique.}

4.1 CADRE GÉNÉRAL DE DESCRIPTION. - Les hypothèses de base de ce modèle viscoplastique [ 9 , $20,24]$ s'inscrivent dans le contexte thermodynamique général des modèles à variables internes [50, 51]. Les diverses extensions, compte tenu des multiples observations expérimentales présentées ci-dessus et du vaste champ des températures étudiées, sont présentées dans ce paragraphe.

On rappelle que, dans le contexte des modèles à variables internes, on peut faire apparaître la dissipation intrinsèque $D$ sous la forme d'une somme des produits des forces thermodynamiques généralisées fonction des variables d'état $A^{k}$ et des flux thermodynamiques $\dot{a}^{k}$, soit $D=\sum_{k} A^{k} \dot{a}^{k}$, et ainsi, vérifier sa positivité traduisant le second principe de la thermodynamique. Le nombre des variables n'est pas fixé mais résulte d'un compromis entre la complexité du modèle et la description des phénomènes observés. On note la souplesse de ce cadre général qui laisse la latitude d'associer à chaque mécanisme identifié de la physique de la déformation, une nouvelle variable interne. Le problème le plus délicat reste le couplage de cette nouvelle variable avec les anciennes.

Dans la présentation du modèle, on s'efforcera de corréler chaque variable à un mécanisme de déformation bien précis, identifié par ailleurs dans des études plus métallurgiques. Evidemment, cette association a toujours un caractère heuristique et peut être sujette à discussion.

4.2 Formulation GÉNÉRALE du MODÈle. - Une présentation succincte des notations est donnée dans l'annexe I et les valeurs numériques des différentes constantes sont reportées dans l'annexe II. 
4.2.1 L'équation viscoplastique d'état. - Il s'agit d'un modèle unifié, on ne considère donc qu'un seul type de déformation inélastique dans l'équation d'état, et de nature purement cinématique [20,51], soit :

$$
\dot{\varepsilon}_{i j}=\frac{3}{2} V_{\mathrm{s}} f(\overline{\sigma-\alpha}) \frac{\sigma_{i j}^{\prime}-\alpha_{i j}^{\prime}}{\overline{\sigma-\alpha}},
$$

avec $f$ fortement non linéaire, ce qui permet de traduire les effets dépendant et quasiment indépendant du temps. On montre [20], que l'on peut accéder à $f$ par la méthode des décréments de contrainte ou de déformation et écrire $f$ sous la forme :

$$
\begin{array}{r}
f(\overline{\sigma-\alpha})=\tanh \left\{\frac{\dot{\varepsilon}_{1}}{V_{\mathrm{s}}} \cdot\left(\frac{N(\bar{\varepsilon}, T)}{\sigma_{0}^{*}}\right)^{n^{*}(T)} \times\right. \\
\left.\times \sinh \left(\frac{\overline{\sigma-\alpha}}{\mathrm{N}(\bar{\varepsilon}, T)}\right)^{n^{*}(T)}\right\},
\end{array}
$$

avec

$$
N(\bar{\varepsilon}, T)=\left(\frac{\dot{\varepsilon}_{1}}{\dot{\varepsilon}_{0}^{*}}\right)^{n_{0}}\left(Y+\beta_{4} Y^{+}\right) .
$$

$\dot{\varepsilon}_{0}^{*}, \quad \sigma_{0}^{*}, \quad n_{0}$ et $\beta_{4}$ sont des constantes, $\dot{\varepsilon}_{1}$ et $n^{*}$ sont deux paramètres dépendant de la température (Eqs. (6) et (7)). L'introduction des fonctions scalaires $Y$ et $Y^{+}$(Eqs. (9) et (10)) décrites ultérieurement, permet d'obtenir conformément aux résultats expérimentaux (Fig. 8), l'augmentation de la composante visqueuse avec la déformation $[7,23$, 51].

La fonction tanh permet de borner à $V_{\mathrm{s}}$ l'évolution de la vitesse d'écoulement lorsque la composante visqueuse devient trop importante. On prendra pour $V_{s}$ la vitesse des bandes de Lüders dans cet acier. Dans l'équation (5a), conformément aux déterminations expérimentales réalisées à l'aide de la méthode des décréments de contrainte durant le fluage, $n$ * dépend de la température (Fig. 16a). On prendra une fonction de la forme (Fig. 16a) :

$$
n^{*}(T)=1+\beta_{0} \exp -\beta_{1}\left(\frac{T-T_{\mathrm{a}}}{T_{\mathrm{f}}-T}\right)^{2},
$$

où $\beta_{0}, \beta_{1}$ sont des constantes, $T_{\mathrm{a}}$ et $T_{\mathrm{f}}$ étant respectivement les températures ambiante $(300 \mathrm{~K})$ et de fusion de l'alliage ( $1696 \mathrm{~K})$. Compte tenu des erreurs inhérentes à la méthode expérimentale, le lissage réalisé à partir de l'équation (6) s'avère suffisant.

$\dot{\varepsilon}_{1}$ est un paramètre qui intègre l'évolution de différentes grandeurs physiques avec la température. En s'inspirant de l'équation adimensionnelle de Dorn [52], on écrira $\dot{\varepsilon}_{1}$ sous la forme :

$$
\dot{\varepsilon}_{1}=A \frac{D_{\text {eff }} G b}{k T} g\left(E_{\gamma}\right) .
$$

Dans cette équation, $A$ est une constante, $G$ est le module de cisaillement, $b$ le vecteur de Burgers, $k$ la constante de Boltzman, $D_{\text {eff }}$ le coefficient de diffusion effectif et $g\left(E_{\gamma}\right)$ une fonction de l'énergie de faute d'empilement $E_{\gamma}$. Le coefficient de diffusion $D_{\text {eff }}$ tient compte des phénomènes de court-circuit de diffusion aux basses températures $[53,54]$ et s'écrit :

$$
D_{\text {eff }}=f_{\mathrm{v}} D_{\mathrm{v}}+f_{\mathrm{c}} D_{\mathrm{c}}
$$

où $D_{\mathrm{v}}$ et $D_{\mathrm{c}}$ sont respectivement, en première approximation, les coefficients de diffusion en volume et sur les courts-circuits de diffusion, du principal constituant de la solution solide. Généralement, $f_{\mathrm{c}}$ n'est pas une constante, mais une fonction de la densité totale des dislocations. On considérera cependant dans un souci de simplification $f_{\mathrm{c}}$ comme constant. La valeur de $\sigma_{0}^{*}$ est directement associée au choix du coefficient de diffusion $D_{\mathrm{v}}$ et l'on prendra celui du fer [20-24] (annexe II).

Dans les aciers inoxydables austénitiques, l'énergie de faute d'empilement $E_{\gamma}$ croît assez fortement avec la température et l'on reporte sur la figure $16 \mathrm{~b}$, d'après Rémy et al. [55], l'évolution de $E_{\gamma}$ pour différents aciers $\mathrm{Fe}-\mathrm{Cr}-\mathrm{Ni}$. On adopte comme fonction de lissage (Fig. 16b);

$E_{\gamma}=E_{0}+\left(E_{\infty}-E_{0}\right)\left(1-\exp -\left(\frac{T}{T_{\mathrm{c}}}\right)^{2}\right)$,

où $E_{0}, E_{\infty}$ et $T_{\mathrm{c}}$ sont des constantes. Les faibles valeurs de $E_{\gamma}$ au voisinage de l'ambiante $\left(E_{\gamma} \simeq 20 \mathrm{~mJ} \cdot \mathrm{m}^{-2}\right.$ pour $T=20^{\circ} \mathrm{C}$ ) peuvent en partie expliquer l'augmentation de la viscosité pour les plus faibles températures étudiées (Figs. 8 et 9). En s'appuyant sur cette interprétation, on écrit $g\left(E_{\gamma}\right)$ sous la forme :

$$
g\left(E_{\gamma}\right)=\exp \left(\frac{E_{\infty}-E_{\gamma}}{E_{\mathrm{c}}}\right),
$$

fonction décroissante de $E_{\gamma}$ et où $E_{\mathrm{c}}$ est une constante. L'évolution de $\dot{\varepsilon}_{1}$ (Eq. (7a)) avec la température est donnée sur la figure $16 \mathrm{c}$ et présente un minimum vers $300{ }^{\circ} \mathrm{C}$, ce qui devrait permettre de décrire les variations de la viscosité avec la température (Figs. 7, 8 et 9).

4.2.2 Description de la variable cinématique $\alpha$. Généralement, les variables cinématiques représentent les contraintes induites par les interactions à différentes distances entre les dislocations mobiles et celles de sous-structure. On distingue classiquement les interactions à longues distances telles que les incompatibilités de déformations plastiques entre grains ou entre différentes phases, les interactions entre précipités et dislocations, ..., et les interactions à moyennes et courtes distances telles que les 
interactions dislocations-dislocations et dislocationsdéfauts ponctuels.

Pour décrire ces différentes échelles d'interaction, on a souvent recours à plusieurs variables cinématiques de cinétiques différentes. C'est cette voie qui est présentement adoptée et illustrée par le système d'équations (8) où il apparaît trois variables cinématiques non linéaires $\underline{\alpha}, \underline{\alpha}_{1}$ et $\underline{\alpha}_{2}[56,57]$, soit :

$$
\begin{aligned}
& \dot{\alpha}_{i j}=p_{\mathrm{m}}\left(2 / 3\left(Y^{*}+Y^{+}\right) \dot{\varepsilon}_{i j}-\right. \\
& \left.-\left(\alpha_{i j}-\alpha_{1 i j}\right) \bar{\varepsilon}\right) \\
& -R_{\mathrm{m}}(T)\left(\operatorname{sh} \beta_{2}(\bar{\alpha})^{M_{1}}\right)^{M_{0}}\left(\frac{\alpha_{i j}}{\bar{\alpha}}\right) \\
& \dot{\alpha}_{1 i j}=p_{1}\left(2 / 3 Y^{*} \dot{\varepsilon}_{i j}-\left(\alpha_{1 i j}-\alpha_{2 i j}\right) \bar{\varepsilon}\right) \\
& \dot{\alpha}_{2 i j}=p_{2}\left(2 / 3 Y^{*} \dot{\varepsilon}_{i j}-\alpha_{2 i j} \bar{\varepsilon}\right),
\end{aligned}
$$

(c)

$p_{\mathrm{m}}, p_{1}, p_{2}, \beta_{2}, M_{0}, M_{1}$ sont des constantes et $R_{\mathrm{m}}(T)$ est thermiquement activée (annexe II).

Plusieurs commentaires s'imposent :

- la variable $\underline{\alpha}$ qui apparaît dans la loi d'écoulement (Eqs. (4) et (5)) permet sensiblement de

avec les valeurs initiales : $\alpha(0)=\alpha_{1}(0)=$ $\alpha_{2}(0)=0$.

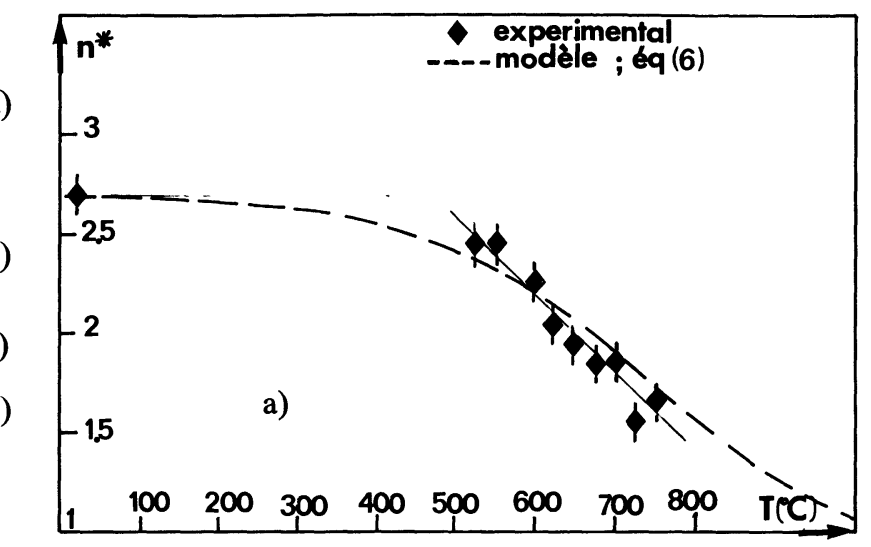

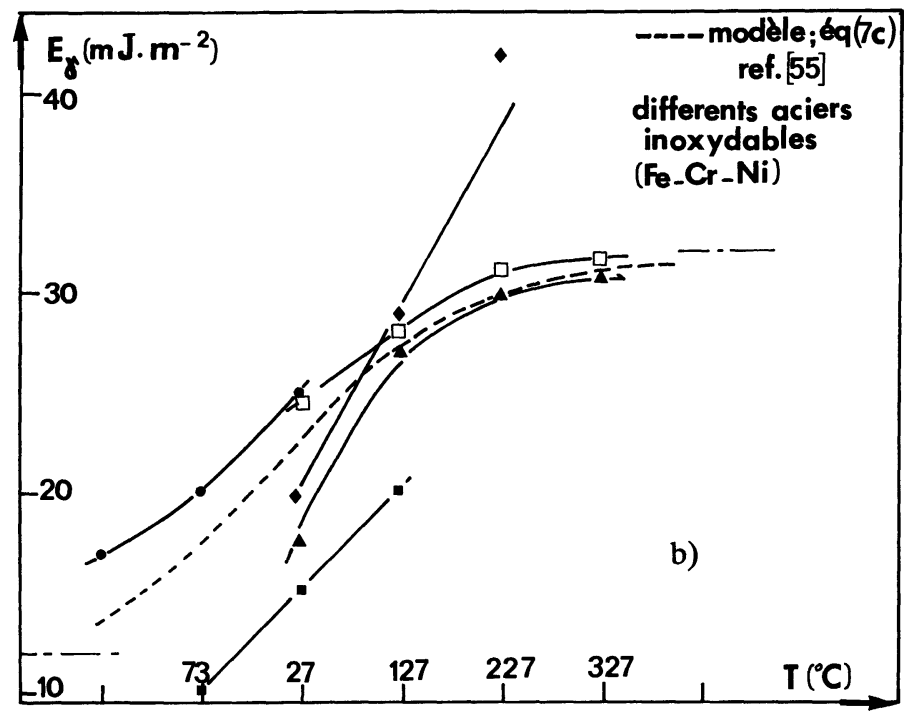

Fig. 16. - (a) Evolution de l'exposant $n^{*}$ avec la température. Essais et fonction de lissage (Eq. (6)). (b) Evolution de l'énergie de faute d'empilement $E_{\gamma}$ avec la température. Résultats expérimentaux [55] et fonction de lissage (Eq. (7c)). (c) Evolution globale de la grandeur, $\dot{\varepsilon}_{1}=A \frac{D_{\text {eff }} G b}{k T} g\left(E_{\gamma}\right)$, avec la température.

[(a) Evolution of the exponent $n^{*}$ with the temperature. Tests and curve fit (Eq. (6)). (b) Evolution of the stacking fault energy $E_{\gamma}$ with the temperature. Experimental results [55] and curve fit (Eq. (7c)). (c) Evolution of the parameter $\dot{\varepsilon}_{1}=A \frac{D_{\text {eff }} G b}{k T} g\left(E_{\gamma}\right)$ with the temperature.]

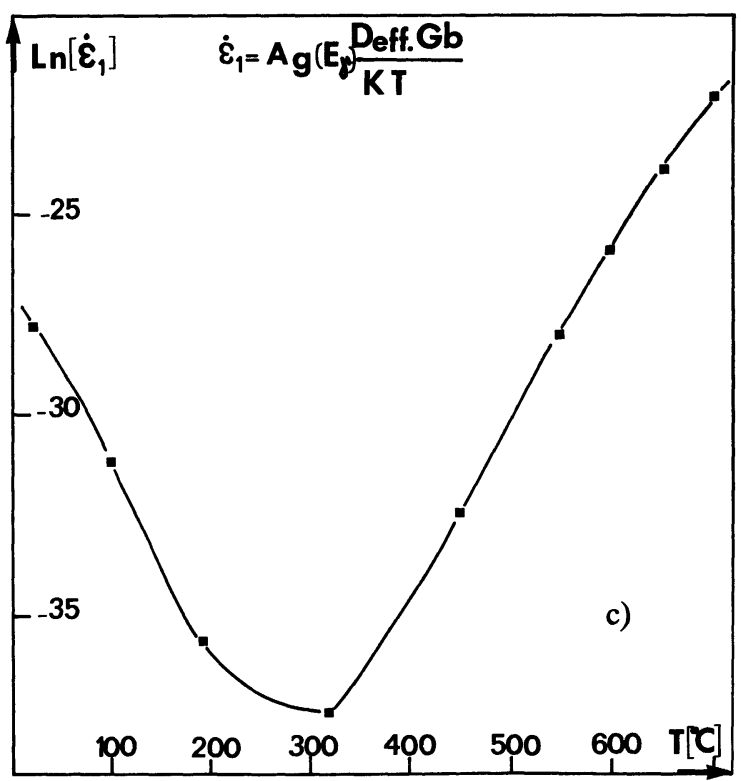


décrire, lorsque $p_{\mathrm{m}}$ est grand, une surface de chargedécharge comme en plasticité classique et les deux variables $\underline{\alpha}_{1}$ et $\underline{\alpha}_{2}$ gèrent l'évolution du centre de cette quasi-surface,

- l'introduction de $\left(Y^{*}+Y^{+}\right)$et $Y^{*}$ (variables scalaires) dans les termes de Prager des cinétiques non linéaires permet de traduire d'une part la croissance des états asymptotiques des variables cinématiques et d'autre part, l'augmentation progressive des modules plastiques tangents, pour une déformation fixée, au cours de la consolidation cyclique [23],

- les cinétiques $\underline{\dot{\alpha}}_{1}$ et $\underline{\dot{\alpha}}_{2}$ (par l'intermédiaire de $p_{1}$ et $p_{2}$ ) permettent d'ajuster parfaitement la courbure des cycles entre déformations imposées et la largeur des boucles entre contraintes imposées,

- en plus de la restauration dynamique traduite par l'adjonction d'un terme de rappel au terme de Prager, il apparaît un membre de restauration statique thermiquement activé $\left(R_{\mathrm{m}}(T)\right)$ dans la loi d'évolution de $\underline{\dot{\alpha}}$. Ce terme permet d'appréhender d'une part l'écoulement stationnaire et d'autre part les phénomènes d'hésitation au fluage lors d'un décrément de contrainte [24],

- en dernier lieu, on peut observer que l'on tend vers une théorie classique de la plasticité indépendante du temps avec surface de charge à évolutions isotrope et cinématique, lorsque dans ce modèle, on fait croître simultanément $n *$ et $p_{\mathrm{m}}$ vers l'infini.

Pour ce qui concerne le rochet $2 \mathrm{D}$ évoqué dans la première partie de l'article, le système d'équations (8) conduit à une très grande surestimation de la déformation progressive. Cependant, on montre clairement [19] que la déformation progressive est due à la normalité de l'écoulement à des surfaces équipotentielles translatées cinématiquement dans l'espace des contraintes. Ainsi, en jouant sur la position des variables cinématiques par l'intermédiaire des termes d'évanescences, on peut faire varier la direction de l'écoulement, donc l'amplitude de la déformation progressive. Dans cette optique, Burlet et Cailletaud [58] ont introduit la notion d'évanescence radiale conduisant à l'accommodation au bout d'un certain nombre de cycles. En suivant cette idée [59], on peut redéfinir la variable cinématique $\alpha_{2 i j}$ qui contrôle l'évolution de $\alpha_{i j}$, sous la forme :

$$
\begin{aligned}
\dot{\alpha}_{2 i j}=p_{2}\left(\frac{2}{3} Y^{*} \dot{\varepsilon}_{i j}-\left(\delta \alpha_{2 i j}+\right.\right. \\
\left.\left.+(1-\delta)\left(\alpha_{2 i j} n_{i j}\right) n_{i j}\right) \bar{\varepsilon}\right) .
\end{aligned}
$$

Avec $\delta=0$, on a une évanescence radiale, pour $\delta=1$, on retrouve l'évanescence normale donnée par l'équation (8c) et dans le cas unidirectionnel (cas de la présente étude), quelle que soit la valeur de $\delta$, on obtient la relation bien connue de l'écrouissage cinématique non linéaire. A l'aide de cette équation, on montre [59] qu'en ajustant le paramètre $\delta$, il est possible de décrire correctement les caractéristiques $\mathrm{du}$ rochet $2 \mathrm{D}$. En ce qui concerne l'ensemble des sollicitations unidirectionnelles ou radiales, les équations (8c) et (8d) sont strictement équivalentes.

4.2.3 Les variables scalaires $Y, Y^{+}$et leur mémorisation. - Compte tenu des développements ultérieurs, si l'on néglige les phénomènes d'interactions avec les défauts ponctuels, on définit $Y^{*}$ par $Y^{*}=Y$ (Eq. (18) avec $y_{\mathrm{c}}=0$ ).

Les variables scalaires sont généralement introduites dans les modèles pour décrire la consolidation ou l'adoucissement cyclique des matériaux et sont directement liées à l'augmentation de la densité des dislocations. L'introduction, dans le présent modèle, de deux variables $Y$ et $Y^{+}$est liée à l'existence de deux familles de dislocations, souvent observées [4] et reflétant schématiquement l'hétérogénéité de distribution des dislocations. On associe $Y$ à la population de dislocation située à l'intérieur des cellules et $Y^{+}$à la population liée aux parois des cellules. Les cinétiques $\dot{Y}$ et $\dot{Y}^{+}$reflètent donc l'augmentation de la densité de ces deux populations qui, par ailleurs, ne présentent pas la même stabilité vis-à-vis des effets de la déformation ou du temps.

Comme nous l'avons évoqué précédemment, les quantités scalaires $Y$ et $Y^{+}$conditionnent l'augmentation des variables cinématiques donc les consolidations cyclique et monotone. Les différentes propriétés cycliques sont donc assujetties à celles de $Y$ et $Y^{+}$, variables essentiellement contrôlées par la déformation plastique cumulée, la température et le temps. On écrira donc :

$$
\begin{aligned}
\dot{Y}=b & \left(Y^{\text {sat }}(T)-Y\right) \times \\
& \times\left[\bar{\varepsilon}-R(T)\left|Y-Y_{0}\right|^{L_{0}} \operatorname{sign}\left(Y-Y_{0}\right)\right]
\end{aligned}
$$

avec :

$$
Y(0)=a_{0}+\gamma_{0} \exp -\left(T / T_{0}\right)^{2},
$$

$Y^{\text {sat }}(T)$ est une variable donnée par l'équation (15),

$$
R(T)=r \frac{D_{\text {eff }} G b}{k T}
$$

et $a_{0}, \gamma_{0}, T_{0}$ et $r$ sont des constantes (voir annexe II).

De la même façon, on écrira pour $\dot{Y}^{+}$:

$$
\begin{gathered}
\dot{Y}^{+}=b^{+}\left(Y^{\text {sat }+}(T)-Y^{+}\right)\left[\dot{q}^{+}-R^{+}(T) \times\right. \\
\left.\left.\times\left|Y^{+}-Y_{0}^{+}\right|^{L_{0}} \operatorname{sign}\left(Y^{+}-Y_{0}^{+}\right)\right]\right\} \\
\quad Y^{+}(0)=0, \\
\text { avec : } \quad Y^{\text {sat }+}=a_{\infty}^{\text {sat }+}+\gamma_{0}^{+} \exp -\left(\frac{T-T^{+}}{T_{0}}\right)^{2},
\end{gathered}
$$


$\dot{q}^{+}$est donnée par l'équation (13), et

$$
R^{+}(T)=r^{+} \frac{D_{\mathrm{eff}} G b}{k T} \quad \text { (voir annexe II) }
$$

On associe à la variable $Y^{+}$le durcissement créé par le développement d'une sous-structure de dislocations telle que celle créée dans les parois des sousgrains ou de la texture de polygonisation. Pour un matériau vierge, cette texture étant absente, on aura bien évidemment $Y^{+}(0)=0$. Les fonctions $Y(0)$ et $Y^{\text {sat }+}$ relatent la décroissance de la densité des différentes populations avec la température.

Dans ces équations, les coefficients $b, b^{+}$fixent la rapidité des consolidations cyclique et monotone, et $Y^{\text {sat }}(T), Y^{\text {sat }+}(T)$ leur amplitude. Il apparaît également des termes de restauration statique qui permettent d'une part de décrire l'écoulement stationnaire et d'autre part, les variations du seuil d'écoulement après vieillissement à haute température, tel que les résultats représentés sur les figures 12 et $13\left(\widetilde{\Delta \sigma}_{\mathrm{r}}\right.$ pour $T \geqslant 550{ }^{\circ} \mathrm{C}$ ). Ces termes de restauration statique reflètent la stabilité vis-à-vis du temps de ces deux populations. On notera que, conformément à l'expérience, seulement une fraction de $Y$ et $Y^{+}$est restaurable, d'où l'introduction des termes $Y_{0}$ et $Y_{0}^{+}$. Ces deux paramètres fixent l'amplitude maximale non restaurable et sont donnés par $[9,24]$ :

$$
\left.\begin{array}{c}
Y_{0}=\operatorname{Max}\left(Y-(1 / 3) \times\left(R_{0} / R\right)^{1 / L_{0}} \times\right. \\
\left.\times\left(\operatorname{sh} \beta_{2}(\operatorname{Max} \bar{\alpha})^{M_{1}}\right)^{M_{0} / L_{0}}\right) \\
Y_{0}^{+}=\operatorname{Max}\left(Y^{+}-\left(R_{0} / R^{+}\right)^{1 / L_{0}} \times\right. \\
\left.\times\left(\operatorname{sh} \beta_{2}(\operatorname{Max} \bar{\alpha})^{M_{1}}\right)^{M_{0} / L_{0}}\right)
\end{array}\right\}
$$

Dans cet acier, l'amplitude de la consolidation cyclique dépend essentiellement de l'amplitude de la déformation imposée (Fig. 4) et le retour à des petits cycles conduit à une évanescence lente mais quasi totale de la mémoire de la plus grande déformation $[23,19,51]$.

On peut voir ici l'intervention de la stabilité vis-àvis de la déformation de ces deux populations. En effet, il est bien connu que la forme et la taille des cellules de polygonisation dépendent de l'amplitude de la déformation mais que, bien qu'il soit facile de développer une fine texture à haut niveau de déformation, il est bien plus difficile de la détruire partiellement pour obtenir une texture plus grossière correspondant à celle qui se serait développée directement pour un niveau de déformation plus faible [4, 52].

Du point de vue de la formulation, en accord avec Chaboche et al. [12], Nouailhas et al. [3] et Ohno [60], on postule, dans l'espace des déformations, l'existence de surfaces de non écrouissage $G$ et $G^{+}$que l'on associera aux variations de $Y^{\text {sat }}$ et $Y^{+}$, soit :

$$
\begin{aligned}
G & =\overline{\varepsilon-\xi}-q \leqslant 0 \\
G^{+} & =\bar{\varepsilon}-q^{*} \leqslant 0
\end{aligned}
$$

avec :

$$
\begin{aligned}
& q^{*}=\int_{0}^{t}\left(2 / 3 \dot{\varepsilon}_{i j} \dot{\varepsilon}_{i j}\right)^{1 / 2} \mathrm{~d} t \\
& \text { déformation plastique cumulée }
\end{aligned}
$$

Les boules $G$ et $G^{+}$ont respectivement pour centre $\xi_{i j}$ et 0 , et pour rayon $q$ et $q^{*}$. A l'exemple des surfaces seuils plastiques, elles ne peuvent évoluer que si les composantes de la déformation $\varepsilon_{i j}$ sont sur leur frontière, ce qui s'exprime par les relations de consistance $G=\dot{G}=0$. A l'intérieur de ces surfaces $q=0$ et $q^{*}=0$. Si $n$ et $n^{*}$ sont respectivement les normales extérieures aux surfaces équipotentielles (espace des contraintes) et à $G$ (espace des déformations), on posera, d'une façon générale $[23,7,61]$ :

$$
\left.\begin{array}{rl}
\dot{q} & =\eta H(G)\left\langle n_{i j} n_{i j}^{*}\right\rangle \overline{\dot{\varepsilon}}-(1-H(G)) g q \overline{\dot{\varepsilon}}, \\
\dot{q}^{+} & =\left(G^{+}\right) \overline{\dot{\varepsilon}}-\left(1-H\left(G^{+}\right)\right) g^{+} q^{+} \overline{\dot{\varepsilon}}
\end{array}\right\}
$$

$\eta, g, g^{+}$sont des constantes, $H($.$) est la fonction$ d'Heaviside et $\langle$.$\rangle les crochets de Macawley (voir$ annexe I). L'équation de consistance $G=\dot{G}=0$, conduit à l'équation d'évolution de $\xi_{i j}$, soit :

$$
\begin{aligned}
\dot{\xi}_{i j}=\sqrt{3} / 2((1-\eta) & H(G)\left\langle n_{i j} n_{i j}^{*}\right\rangle n_{i j}^{*} \overline{\dot{\varepsilon}}+ \\
+ & \left.(1-H(G)) n_{i j}^{*} g q \overline{\dot{\varepsilon}}\right) .
\end{aligned}
$$

Les mémorisations des prédéformations cyclique et monotone sont effectuées par les premiers termes de gauche des équations (13) et l'évanescence lente de ces mémoires par les seconds termes. Le paramètre $\eta$ est toujours compris entre 0 et 1 . Si $\eta=1$, la mémoire est établie au premier quart de cycle ( $\eta=0,5$ à $3 / 4$ cycle), alors que $\eta \leqslant 0,5$ produit une certaine progressivité dans l'établissement de celleci [60]. L'ajustement du modèle aux courbes cyclique et monotone conduit à $\eta=3 \times 10^{-2}$.

A l'inverse, le retour progressif de la mémoire au niveau de la plus faible déformation s'effectue exponentiellement avec la déformation cumulée, la rapidité étant fixée par les paramètres $g$ et $g^{+}$.

Il reste à relier l'évolution de $Y^{\text {sat }}$ à $q, \dot{Y}^{+}$étant directement lié à $\dot{q}^{+}$dans l'équation (10). On écrit :

$$
\left.\begin{array}{l}
\dot{Y}^{\text {sat }}=b^{\text {sat }}\left(Y_{\infty}^{\text {sat }}(T)-Y^{\text {sat }}\right) \dot{q} \text { avec } \\
Y_{\infty}^{\text {sat }}=a_{\infty}^{\text {sat }}+\gamma_{0} \exp -\left(T / T_{0}\right)^{2}, \\
Y^{\text {sat }}(0)=\left\{a_{0}^{\text {sat }}+\gamma_{0} \exp -\left(T / T_{0}\right)^{2}\right\} \times \\
\times\left(1+\gamma_{\mathrm{c}} y_{\mathrm{c}}\right)
\end{array}\right\}
$$


$a_{\infty}^{\text {sat }}, a_{0}^{\text {sat }}, b^{\text {sat }}$ et $T_{0} \quad$ sont $\quad$ des constantes (voir annexe II).

Les fonctions $Y_{\infty}^{\text {sat }}$ et $Y^{\text {sat }}(0)$ reflètent la décroissance des différents paramètres de sous-structure avec la température (diamètre des cellules de polygonisation...), affectée par la fonction d'interaction dislocations-défauts ponctuels résultant du temps de maintien en température.

En résumé, à l'aide des équations (4) à (15), il est possible de décrire, hormis les interactions dislocations-défauts ponctuels que nous évoquerons ci-dessous, l'essentiel des propriétés monotones et cycliques relatives aux incréments de déformation imposée puis au retour à des petits cycles, en partie aux effets de la température sur le cycle stabilisé et à la sensibilité positive de la contrainte stabilisée à la vitesse de déformation. L'effet de rochet $2 \mathrm{D}$ de traction-torsion peut être également décrit en remplaçant l'équation (8c) par (8d).

4.2.4 Formulation des variables d'interaction dislocations - défauts ponctuels. - Comme nous l'avons démontré précédemment, il existe une très forte interaction dislocations-amas de défauts ponctuels pour les températures intermédiaires $(100 \leqslant T \leqslant$ $500{ }^{\circ} \mathrm{C}$ ).

A notre connaissance, l'approche phénoménologique de la modélisation de ces interactions et son introduction dans un modèle de comportement n'a fait l'objet que de très peu d'étude $[12,51,62]$. Nous proposons et explicitons une formulation directement issue des observations expérimentales décrites précédemment, à savoir que l'on assiste à un durcissement par le temps (Eqs. (1) à (3)) mais évanescent avec la déformation (Figs. 11, 12). Ainsi, dans le contexte des modèles à variable interne, on attribuera à chaque interaction $(i)$ une composante scalaire $Y^{(i)}$, telle que :

$$
\left.\begin{array}{cc} 
& \dot{Y}^{(i)}=-p^{(i)} Y^{(i)} \overline{\dot{\varepsilon}}+\frac{Y_{\infty}^{(i)}-Y^{(i)}}{\tau^{(i)}} \\
\text { avec } & \tau^{(i)}=\tau_{0}^{(i)} \exp \frac{W^{(i)}}{k T} \\
\text { et } & Y_{\infty}^{(i)}=Y_{\infty}^{(i) \text { sat }} \exp -\left(\frac{T-T^{(i)}}{A^{(i)}}\right)^{2} \cdot
\end{array}\right\}
$$

Dans ces équations, $p^{(i)}$ est une constante, $\tau^{(i)}$ le temps de relaxation thermiquement activé intrinsèque de l'interaction considérée (Eq. (2)) et $Y_{\infty}^{(i)}$ sa fonction d'efficacité que nous prendrons, en accord avec quelques auteurs [12, 62-64], sous la forme d'une répartition Gaussienne (distribution Log-Normale). D'autres types de distribution auraient pu être choisies [34]. $\tau_{0}^{(i)}, W^{(i)}, Y_{\infty}^{(i) \text { sat }}$, $T^{(i)}$ et $A^{(i)}$ sont des constantes directement accessibles par l'expérience et dont les valeurs sont reportées dans l'annexe II.
L'équation différentielle (16) prévoit bien un durcissement par le temps (second terme de droite) exponentiellement évanescent avec la déformation (premier terme de droite). Le régime d'équilibre, $\dot{Y}^{(i)}=0$, correspond à :

$$
Y^{(i) \mathrm{st}}=\frac{Y_{\infty}^{(i)}(T)}{1+\tau^{(i)} p^{(i)} \dot{\bar{\varepsilon}}} .
$$

$Y^{(i) s t}$ est une fonction décroissante de la vitesse et offre ainsi, comme nous le montrons ultérieurement, une possibilité de description de la valeur négative de l'exposant de sensibilité de la contrainte à la vitesse (Figs. 2, 5 et 6). Les cinq interactions dénombrées étant liées à la redistribution spatiale des dipôles ou des amas de défauts ponctuels dans le champ de contrainte des dislocations, donc fonction de la distribution et de la densité de ces dernières, il reste à relier le spectre d'interaction à la variable scalaire $Y$ qui quantifie l'évolution de la densité des dislocations mobiles. On considère que les interactions avec les dislocations de sous-structures caractérisées par $Y^{+}$sont négligeables. Ainsi, on définit une nouvelle variable scalaire $Y^{*}$, telle que :

$$
Y^{*}=Y\left(1+\gamma_{\mathrm{c}} y_{\mathrm{c}}\right) \text { avec } y_{\mathrm{c}}=\frac{\sum_{(i)} Y^{(i)}}{\sum_{(i)} Y_{\infty}^{(i) \mathrm{sat}}}
$$

et $i \in\{1,5\}$,

où $y_{\mathrm{c}}$ est la fraction totale d'atomes réorientés à courte distance et $\gamma_{\mathrm{c}}$ une constante de répartition. On retrouve, dans la fraction $y_{\mathrm{c}}$, la forme additive formulée dans l'équation (1). A ce stade, on peut donc décrire l'augmentation du durcissement supplémentaire avec la déformation (Figs. 12, 13), ainsi que les effets de vitesse (Figs. 2 et 5). En effet, dans le domaine des grandes vitesses et en dehors des températures d'interactions, on a bien évidemment $Y^{*}=Y\left(y_{\mathrm{c}}=0\right)$ et pour les très faibles vitesses, ou les périodes de vieillissement $\left(\dot{\varepsilon}_{\mathrm{t}}=0\right)$, le spectre est maximum et correspond à

$$
y_{\mathrm{c}}=\sum_{(i)} Y_{\infty}^{(i)} / \sum_{(i)} Y_{\infty}^{(i) \mathrm{sat}} .
$$

\section{Possibilités du modèle, identification et simulations numériques.}

L'identification du modèle a été réalisée sur la plage 20-700 ${ }^{\circ} \mathrm{C}$ et l'ensemble des coefficients et paramètres sont donnés dans l'annexe II. Afin de guider le lecteur dans la démarche d'identification du modèle, d'une part, nous rappelons succinctement les méthodes expérimentales pour la détermination des para- 
mètres fixes et d'autre part, nous précisons les paramètres libres fixant les degrés de liberté de l'ensemble du modèle.

Pour ce qui concerne l'équation d'état (Eqs. (4) à (7d)), sa forme et l'ensemble des paramètres y figurant sont déterminés expérimentalement à partir de la méthode des décréments de contrainte ou de déformation respectivement, pour différentes températures, durant des essais de fluage ou de traction à vitesse imposée [20, 24]. En effet, par ces techniques, on accède directement à la composante visqueuse : $\tilde{\sigma}_{\mathrm{v}}=\tilde{\sigma}-\tilde{\alpha}$, ce qui permet d'obtenir la relation : $\overline{\dot{\varepsilon}}=f|\sigma-\alpha|$, d'où la détermination de $n^{*}$ dont l'évolution avec la température est approximée par l'équation (6). Pour les plus hautes températures, lorsque le fluage est conséquent $\left(T>550{ }^{\circ} \mathrm{C}\right.$, $g\left(E_{\gamma}\right)=1$ et $\left.D_{\text {eff }}=D_{\mathrm{v}} \simeq D_{\mathrm{Fe}}\right)$, l'obtention d'une courbe maîtresse de Dorn : $\frac{\dot{\varepsilon} k T}{D_{\mathrm{Fe}} G b}=f\left(\frac{\sigma_{\mathrm{v}}}{\sigma_{0}^{*}}\right)^{n^{*}}$, permet d'accéder aux paramètres $A$ et $\sigma_{0}^{*}$.

Comme nous l'avons mentionné précédemment, pour les températures intermédiaires $(200 \leqslant T \leqslant$ $500{ }^{\circ} \mathrm{C}$ ), le fluage est quasi inexistant alors qu'il réapparaît au voisinage de l'ambiante $\left(20 \leqslant T \leqslant 150^{\circ}\right)$. Le coefficient de diffusion : $D_{\text {eff }} \simeq f_{\mathrm{c}} D_{\mathrm{c}}$ étant très faible, ce comportement ne peut s'expliquer que par la faible valeur de l'énergie de faute d'empilement $E_{\gamma}$ à la température ambiante, en d'autres termes que $\dot{\varepsilon}_{1}$ (Eq. (7a)), via la fonction $g\left(E_{\gamma}\right)$ (Eq. (7d)), doit être élevée vers l'ambiante, ce qui justifie le choix de la forme exponentielle pour $g\left(E_{\gamma}\right)$. L'évolution de $E_{\gamma}$ est approximée par l'équation (7c) et la fonction $g\left(E_{\gamma}\right)$, via le paramètre $E_{\mathrm{c}}$, est ajustée numériquement sur les courbes de fluage à 20 et $100{ }^{\circ} \mathrm{C}$. Pour les essais à vitesse imposée, la connaissance expérimentale de l'évolution de la composante visqueuse $\tilde{\sigma}_{v}$ avec la température et la déformation (Fig. 8) permet de déterminer, moyennant l'identification des fonctions scalaires $Y$ et $Y^{+}$, les paramètres $\dot{\varepsilon}_{0}^{*}, n_{0}$ et $\beta_{4}$ (Eq. (5b)).

De nombreux faits expérimentaux plaident en faveur d'un modèle purement cinématique $[20,24$, 51]. Cependant, le nombre de variables cinématiques, que l'on a pris égal à trois, reste tout à fait subjectif. A moindre échelle, on se retrouve dans une situation analogue à celle des modèles multicouches où il n'est pas rare de dénombrer une dizaine de termes cinématiques linéaires. Compte tenu du couplage entre les variables cinématiques et les fonctions scalaires, l'identification des paramètres des équations (8) à (18) s'effectue simultanément. Lorsque l'on néglige d'une part les termes de restauration statique et d'autre part, les termes d'interaction à courte distance $\left(y_{\mathrm{c}}=0\right)$, on peut montrer que la courbe de consolidation cyclique s'exprime par :

$$
\left.\begin{array}{l}
\frac{\Delta \sigma^{\text {stab }}}{2}-\sigma_{\mathrm{v}}= \\
=Y^{\text {sat }}\left[1+\tanh p_{1} \frac{\Delta \varepsilon^{p}}{2}+\tanh p_{2} \frac{\Delta \varepsilon^{p}}{2}\right] \\
\text { avec } \\
Y^{\text {sat }}=Y_{\infty}^{\text {sat }}+\left(Y^{\text {sat }}(0)-Y_{\infty}^{\text {sat }}\right) \exp b^{\text {sat }} \frac{\Delta \varepsilon^{p}}{2} .
\end{array}\right\}
$$

L'équation (19) est obtenue avec $p_{\mathrm{m}}$ grand et $Y^{+}$négligeable lorsque $\Delta \varepsilon^{p} / 2$ est petit. Pour une isotherme fixée, la connaissance de la courbe de consolidation cyclique et de la composante visqueuse permet d'ajuster les paramètres $Y_{\infty}^{\text {sat }}, Y^{\text {sat }}(0), b^{\text {sat }}$, $p_{1}$ et $p_{2}$. Notons que les valeurs des coefficients $p_{\mathrm{m}}, p_{1}$ et $p_{2}$ doivent également être compatibles avec la courbure des boucles stabilisées. L'analyse du premier quart de cycles $\left(Y^{\text {sat }} \simeq Y^{\text {sat }}(0)\right)$ en fonction de l'amplitude de la déformation permet d'ajuster la valeur initiale de $Y$, soit $Y(0)$, et la rapidité de l'établissement du cycle stabilisé les paramètres $b$ et $\eta$. En toute rigueur, l'étude de l'effet d'un écrouissage sur le comportement cyclique permettrait d'ajuster exactement le paramètre de mémorisation $\eta$. Le comportement cyclique tant identifié, on s'attache ensuite à décrire les courbes monotones. Le manque de durcissement observé sitôt que l'on dépasse quelques pourcents est comblé par la fonction $Y^{+}$, d'où l'ajustement des paramètres $b^{+}$et $Y_{\infty}^{\text {sat }+}$. On prend $\eta^{+}=1$ car il ne faut pas que cette fonction vienne perturber le comportement cyclique déjà identifié. Ce type d'identification étant réalisée pour différentes isothermes, on accède donc à l'évolution des grandeurs $Y(0), Y_{\infty}^{\text {sat }+}, Y^{\text {sat }}(0)$ et $Y_{\infty}^{\text {sat }}$ avec la température, d'où les différentes fonctions de lissage mentionnées précédemment.

Les différents termes de restauration sont ajustés d'une part à partir de l'analyse de l'écoulement stationnaire et d'autre part à partir d'essais de restauration statique sous contrainte nulle $[9,20$, 24]. La dépendance, pour différentes isothermes, de la vitesse stationnaire avec la contrainte interne $\bar{\alpha}$, permet de déterminer les paramètres $\beta_{2}, M_{1}$, $M_{0}$ et $R_{0}(T)$. L'étude du phénomène d'hésitation au fluage consécutivement à un décrément de contrainte conduit à l'ajustement de $R_{\mathrm{m}}(T)=R_{0}(T) / \beta_{3}$ et de $p_{\mathrm{m}}$. En dernier lieu, l'indifférence de l'écoulement stationnaire à l'histoire du chargement en contrainte et l'étude expérimentale de la restauration du seuil d'écoulement sous contrainte nulle (mesure de $Y_{0}+Y_{0}^{+}$) permet d'obtenir les paramètres $L_{0}$, $R(T)$ et $R^{+}(T)$.

Si l'on tient compte des phénomènes d'interactions avec les défauts ponctuels, la partie la plus délicate reste l'ajustement du spectre d'interaction $\left(1+\gamma_{c} y_{c}\right)$ par l'intermédiaire des fonctions d'efficacité. Celui-ci s'effectue simultanément à partir des 
courbes cinétiques reportées sur la figure 14 (amplitude des oscillations), de la courbe $\widetilde{\Delta \sigma}_{\mathrm{r}}(>0)=$ $f(T)$ (Fig. 12) ce qui permet de calculer la fraction précipité maximale ( $y_{\mathrm{c}}$ lorsque $\dot{\varepsilon}^{\mathrm{T}} \rightarrow 0$ ), et de l'amplitude de la bosse obtenue sur l'évolution du cycle stabilisé en fonction de la température pour une vitesse fixée (Fig. 3). La méthode n'est pas analytique mais procède par calculs successifs et permet d'accéder aux paramètres $Y_{\infty}^{(i) \text { sat },} T^{(i),} A^{(i)}$ et $\gamma_{\mathrm{c}}$. Ainsi, on présente figure 17, la forme du spectre d'interaction obtenue à l'aide des équations (16), (17), (18). On remarque, à haute température, la présence d'une interaction supplémentaire

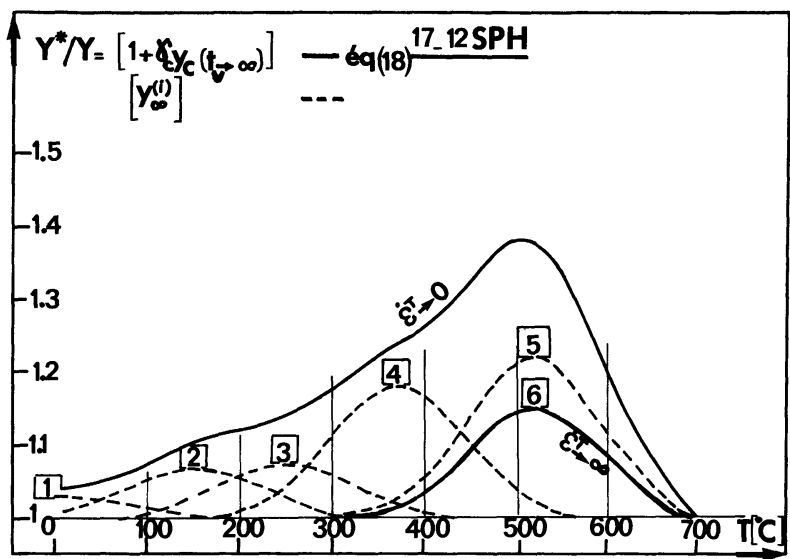

Fig. 17. - Calcul du spectre d'interaction, $Y^{*} / Y=\left(1+\gamma_{\mathrm{c}} y_{\mathrm{c}}\right)$ (Eq. (18)).

[Calculation of the interaction spectrum, $Y^{*} / Y=\left(1+\gamma_{\mathrm{c}} y_{\mathrm{c}}\right)$ (Eq. (18)).] $((i)=6)$, homothétique à l'interaction $(i)=5$, et qui représente le résidu des interactions dont le temps de relaxation est trop court pour être accessible à l'expérience $\left(\tau^{(i)}<0,5 \mathrm{~s}\right)$, ce qui correspond sensiblement à une réorientation spontanée. On écrira donc, $Y^{(6)}=Y_{\infty}^{(6)}(T)$. Du point de vue de l'identification, cette nouvelle interaction permet de limiter la décroissance de la contrainte avec la vitesse (Figs. 2 et 5 ).

Sur la figure 1 on a tracé l'estimation de la contrainte d'écoulement, pour différents niveaux de déformation, obtenue avec le modèle complet. De même sur la figure 3, on représente l'estimation de la contrainte stabilisée en fonction de la température, pour une amplitude fixée, et à $600{ }^{\circ} \mathrm{C}$ (Figs. 3 et 4) pour les quatre amplitudes testées.

La figure 18 représente l'estimation de la vitesse de la consolidation cyclique sur la plage de températures étudiée.

L'accord global, propriétés monotones et cycliques confondues, est satisfaisant.

L'une des possibilités nouvelle et remarquable est la description de l'évolution du coefficient de sensibilité à la vitesse $n$, avec la température, aussi bien sous sollicitations monotones (Fig. 2) que cycliques (Figs. 5 et 6). Conformément à l'expérience, $n$ est positif à basses et hautes températures et négatif aux températures intermédiaires. En ce qui concerne les propriétés visqueuses, on représente figure 8 , l'estimation de la composante visqueuse fonction de la déformation et de la température. Compte tenu de la dispersion expérimentale et des incertitudes liées à la technique de mesure, l'accord peut être jugé satisfaisant d'autant plus que les paramètres du modèle ont été directement ajustés sur les courbes

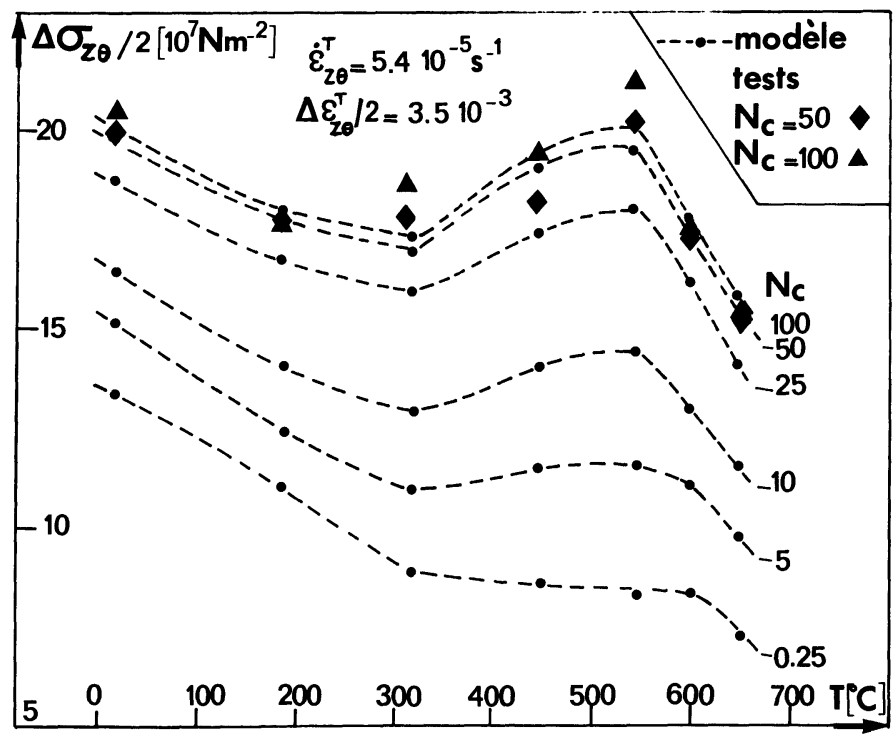

Fig. 18. - Evaluation de la vitesse de la consolidation cyclique sur la plage de température étudiée.

[Evaluation of the rate of cyclic hardening in the temperature domain under study.] 


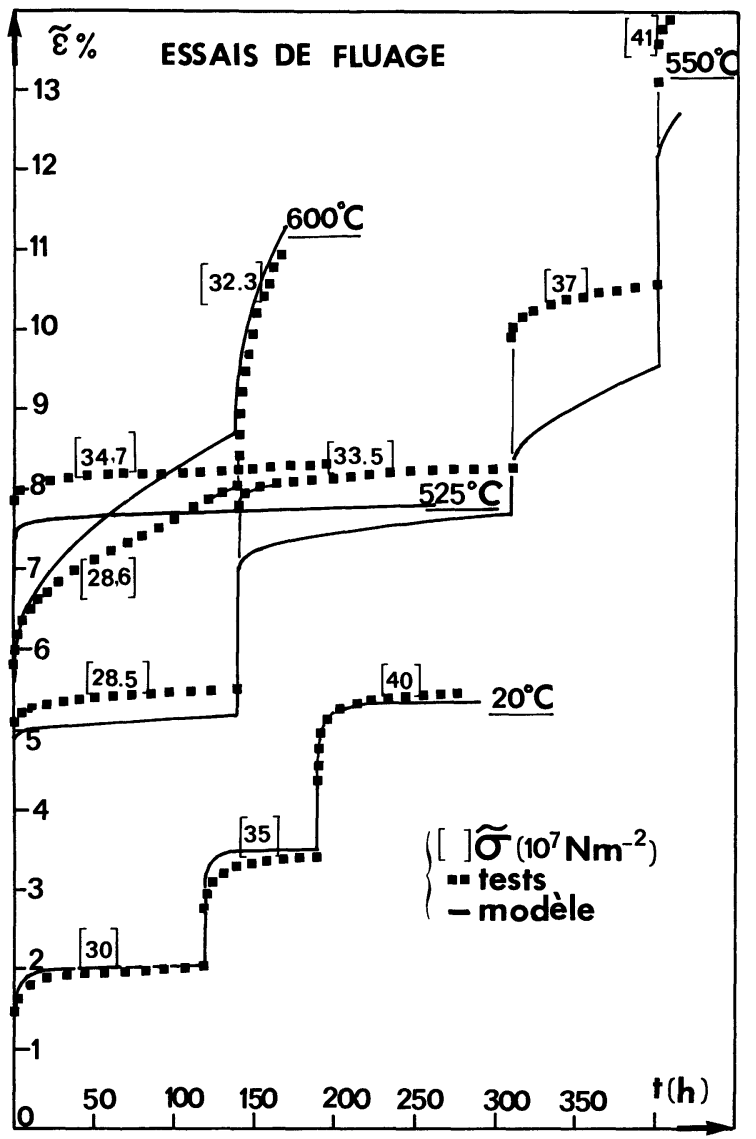

Fig. 19. - Simulation de différents essais de fluage sous niveaux croissants de contrainte et pour différentes températures.

[Simulation of different creep tests under increasing levels of stress and for different temperatures.]

de fluage, dont un exemple est fourni sur la figure 19, et de relaxation à différentes températures $[9,63]$.

En dernier lieu, la figure 20 montre un exemple de restitution du phénomène de retour de limite d'élasticité, consécutivement à une relaxation de $48 \mathrm{~h}$ à $320{ }^{\circ} \mathrm{C}$ pour une déformation $\tilde{\varepsilon}=20 \%$. On signale que seuls quelques points de la figure 12 ont été calculés et que l'accord quantitatif est bon. Comme nous l'avons mentionné précédemment, la partie $\widetilde{\Delta \sigma}_{\mathrm{r}}<0$ est assurée par la présence de termes de restauration sur certaines variables $[9,24,57]$. Pour ce qui concerne les essais cycliques multiaxés non radiaux, les problèmes paraissent résolus, quelle que soit la température $[59,65,66]$ et en particulier dans le cas du rochet $2 \mathrm{D}$, en prenant $\delta$ fonction décroissante de la température (Eq. (8d)), il semble possible d'appréhender l'évolution de la vitesse de la déformation progressive avec le nombre de cycle et la température (Fig. 7). Le dernier problème ouvert reste la modélisation du rochet $1 \mathrm{D}$, bien que plu-

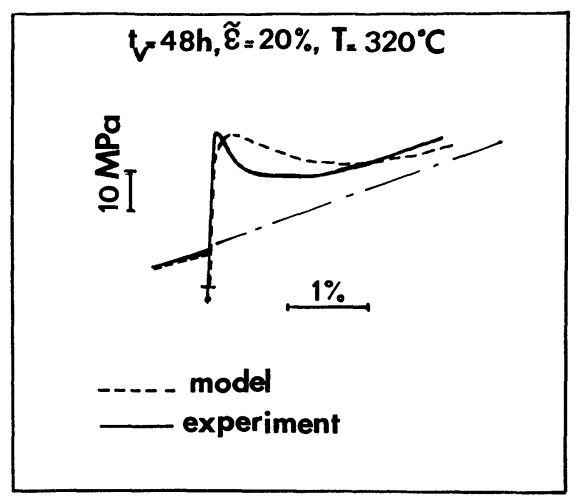

Fig. 20. - Exemple de modélisation de retour de limite d'élasticité.

[Modelization example of the yield point return.]

sieurs améliorations notoires aient été apportées [19, $67,68]$. Ce sujet ne sera donc pas abordé dans le présent article.

\section{Conclusions.}

Aux températures intermédiaires, une composante importante des propriétés monotones, cycliques et visqueuses de cet acier inoxydable austénitique est due à la présence d'interaction entre dislocations et défauts ponctuels. Le mécanisme d'interaction est certainement, pour un réseau C.F.C., la réorientation à courte distance de dipôles dans le champ de contrainte des dislocations. Les atomes de carbone et d'azote sont nécessaires à la formation de ces dipôles ou autres configurations plus complexes. Pour cet acier, on dénombre au moins cinq interactions. On propose une formulation phénoménologique, qui, intégrée dans le contexte d'un modèle viscoplastique unifié formulé pour une plage étendue de températures, permet de décrire la grande variété des comportements observés sur cet acier entre 20 et $700{ }^{\circ} \mathrm{C}$.

A ce stade de la modélisation et de l'identification, il ne reste plus qu'à confronter les prévisions du modèle à de vrais essais anisothermes où sollicitations mécaniques et thermiques évoluent simultanément. Cette phase ultime est en cours de réalisation.

\section{Remerciements.}

- Cette étude a été financée par Electricité de France (EDF) sous le contrat $\mathrm{n}^{\circ} 3 \mathrm{~K} 0665$.

- Une communication sous forme condensée de cet article a été présentée au XIX Canadian Fracture Conference à Ottawa (Canada, 1989). 


\section{Annexe I}

\section{Nomenclature succincte}

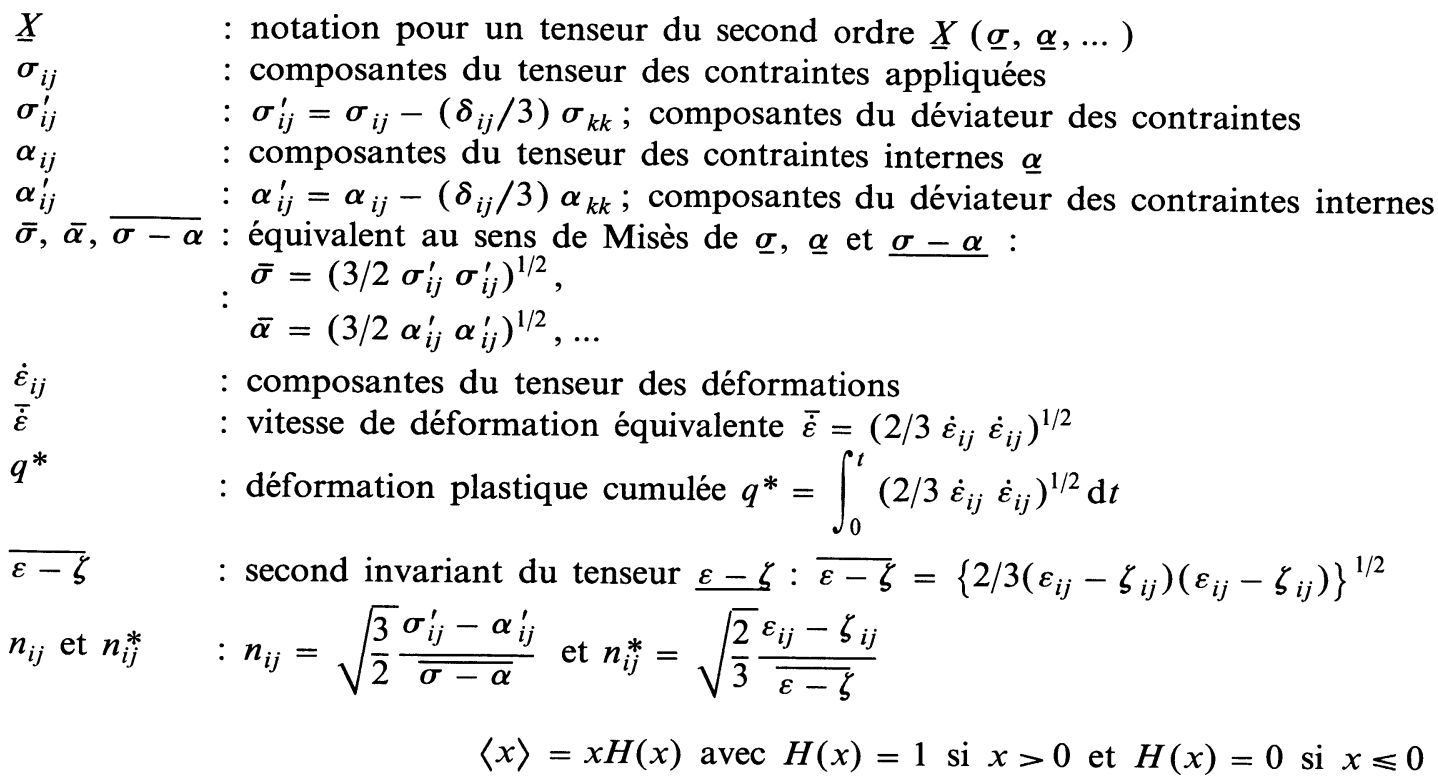

T : température

\section{Fonctions, paramètres et constantes du modèle}

$\rightarrow$ Constantes physiques de l'équation d'état :

- Coefficients de diffusion :

$$
\begin{gathered}
D_{\mathrm{Fe}}(\mathrm{v})=D_{0 \mathrm{Fe}}(\mathrm{v}) \exp -\frac{Q_{\mathrm{v}}}{k T} \text { avec } D_{0 \mathrm{Fe}}(\mathrm{v})=1,74 \times 10^{-4} \mathrm{~m}^{2} \mathrm{~s}^{-1} \text { et } Q_{\mathrm{v}}=2,98 \mathrm{eV} \\
D_{\mathrm{Fe}}(\mathrm{c})=D_{0 \mathrm{Fe}}(\mathrm{c}) \exp -\frac{Q_{\mathrm{c}}}{k T} \text { avec } D_{0 \mathrm{Fe}}(\mathrm{c})=3,1 \times 10^{-5} \mathrm{~m}^{2} \mathrm{~s}^{-1} \text { et } Q_{\mathrm{c}}=1,87 \mathrm{eV} \\
D_{\mathrm{eff}}=f_{\mathrm{v}} D_{\mathrm{Fe}}(\mathrm{v})+f_{\mathrm{c}} D_{\mathrm{Fe}}(\mathrm{c}) \text { avec } f_{\mathrm{v}}=1 \text { et } f_{\mathrm{c}}=1 \times 10^{-8} .
\end{gathered}
$$

- Module de cisaillement :

$$
G=7,7 \times 10^{10}\left(1-4,7 \times 10^{-4}(T-300)\right)\left(\mathrm{Nm}^{-2}\right) .
$$

- Constantes physiques :

$$
b=2,5 \times 10^{-10} \mathrm{~m}, T_{\mathrm{F}}=1696 \mathrm{~K} \text { et } V_{\mathrm{s}}=1 \mathrm{~s}^{-1} .
$$

- Paramètres du modèle :

$$
A=4,32 \times 10^{-12} \text { et } \sigma_{0}^{*}=1,5 \times 10^{5} \mathrm{Nm}^{-2} .
$$

- Coefficient de sensibilité de la vitesse à la composante visqueuse :

$$
n^{*}=1+\beta_{0} \exp -\beta_{1}\left(\frac{T-T_{\mathrm{a}}}{T_{\mathrm{F}}-T}\right)^{2} \text { avec } \beta_{0}=1,7, \beta_{1}=0,7 \text { et } T_{\mathrm{a}}=300 \mathrm{~K} \text {. }
$$

- Fonction de variation de l'énergie de faute d'empilement :

$$
g\left(E_{\gamma}\right)=\exp \left[\frac{E_{\infty}-E_{0}}{E_{\mathrm{c}}} \exp -\left(\frac{T}{T_{\mathrm{c}}}\right)^{2}\right] \text { avec }\left\{\begin{array}{l}
T_{\mathrm{c}}=340 \mathrm{~K}, E_{\infty}=32 \mathrm{~mJ} . \mathrm{m}^{-2} \\
E_{0}=12 \mathrm{~mJ} \cdot \mathrm{m}^{-2} \text { et } E_{\mathrm{c}}=0,185 \mathrm{~mJ} . \mathrm{m}^{2}
\end{array}\right.
$$


$\rightarrow$ Constantes physiques et paramètres des variables d'interactions $Y^{(i)}$ :

\begin{tabular}{|c|c|c|c|c|c|c|}
\hline$(i)$ & $(1)$ & $(2)$ & $(3)$ & $(4)$ & $(5)$ & $(6)$ \\
\hline$W_{(i)(\mathrm{eV})}$ & 0,95 & 0,98 & 1,22 & 1,18 & 1,25 & - \\
$\tau_{0}^{(i)}(\mathrm{s})$ & $1,6 \times 10^{-11}$ & $1,1 \times 10^{-9}$ & $4,3 \times 10^{-10}$ & $5,4 \times 10^{-8}$ & $3,6 \times 10^{-7}$ & - \\
$T^{(i)}(\mathrm{K})$ & 293 & 423 & 523 & 643 & 793 & 793 \\
$A^{(i)}(\mathrm{K})$ & 100 & 100 & 100 & 100 & 100 & 100 \\
$p^{(i)}$ & 100 & 100 & 100 & 100 & 100 & - \\
$Y_{\left(10^{(i)} \mathrm{Nm}^{-2}\right)}^{(00)}$ & 0,03 & 0,07 & 0,075 & 0,18 & 0,22 & 0,15 \\
\hline$\sum Y_{\infty}^{(i) \mathrm{sat}^{-}}=0,725 \times 10^{7} \mathrm{Nm}^{-2}, \gamma_{\mathrm{c}}=0,72$ \\
\hline
\end{tabular}

$\rightarrow$ Fonctions et constantes des lois isotropes :

$Y_{(0)}^{\text {sat }}=a_{0}^{\text {sat }}+\gamma_{0} \exp -\left(\frac{T}{T_{0}}\right)^{2}$

$Y(0)=a_{0}+\gamma_{0} \exp -\left(\frac{T}{T_{0}}\right)^{2}$

$Y_{\infty}^{\text {sat }}=a_{\infty}^{\text {sat }}+\gamma_{0} \exp -\left(\frac{T}{T_{0}}\right)^{2}$

$Y^{\text {sat }+}=a_{\infty}^{\text {sat }+}+\gamma_{0}^{+} \exp -\left(\frac{T-T^{+}}{T_{0}}\right)^{2}$

avec $\left\{\begin{array}{l}\mathrm{a}_{0}^{\text {sat }}=6,3 \times 10^{7} \mathrm{Nm}^{-2}, a_{0}=3,5 \times 10^{7} \mathrm{Nm}^{-2}, \\ a_{\infty}^{\text {sat }}=12,7 \times 10^{7} \mathrm{Nm}^{-2}, a_{\infty}^{\text {sat }+}=17 \times 10^{7} \mathrm{Nm}^{-2}, \\ \gamma_{0}=6,1 \times 10^{7} \mathrm{Nm}^{-2}, \gamma_{0}^{+}=17,6 \times 10^{7} \mathrm{Nm}^{-2} \text { et } \\ T_{0}=453 \mathrm{~K}, T^{+}=173 \mathrm{~K}\end{array}\right.$

$b=8,5$ et $b^{+}=10$

$L_{0}=3,5$

$R_{0}=\beta_{3} R_{\mathrm{m}}$ avec $\beta_{3}=5,13 \times 10^{-12}$

$R^{+}=r^{+} \frac{D_{\text {eff }} G b}{k T}, R=r \frac{D_{\text {eff }} G b}{k T}$ tel que :

$$
\left(\frac{1}{R^{+}}\right)^{1 / L_{0}}+\left(\frac{1}{R}\right)^{1 / L_{0}}=\left(\frac{1}{R^{*}}\right)^{1 / L_{0}} \text { avec } \rightarrow R^{*}=r^{*} \frac{D_{\text {eff }} G b}{k T}
$$

et $r^{*}=6 \times 10^{-34}\left(\mathrm{Nm}^{-2}\right)^{-3,5} \mathrm{~s}^{-1}$.

$\rightarrow$ Constantes de mémorisation des prédéformations :

$$
\begin{aligned}
\eta & =3 \times 10^{-2} \\
g & =g^{+}=0 \\
b^{\text {sat }} & =120
\end{aligned}
$$

$\rightarrow$ Constantes des lois cinématiques :

$$
\begin{aligned}
& p_{\mathrm{m}}=10^{4}, p_{1}=1800 \text { et } p_{2}=350 \\
& \delta=2 \times 10^{-2} \text { pour } T=600{ }^{\circ} \mathrm{C} \\
& M_{1}=2, M_{0}=4 \\
& R_{\mathrm{m}}=R_{\mathrm{m} 0} \exp -\frac{\Delta H_{0}}{k T} \text { avec } R_{\mathrm{m} 0}=1,58 \times 10^{35} \mathrm{Nm}^{-2} \mathrm{~s}^{-1} \text { et } \Delta H_{0}=5,6 \mathrm{eV} \\
& \beta_{2}=1,6 \times 10^{-7}\left(\mathrm{Nm}^{-2}\right)^{-2} .
\end{aligned}
$$

$\rightarrow$ Constantes affectant la composante visqueuse :

$\dot{\varepsilon}_{0}^{*}=2,56 \times 10^{-6} \mathrm{~s}^{-1}, n_{0}=0,137$ et $\beta_{4}=0.5$. 


\section{Bibliographie}

[1] Lieurade H. P., Rabbe P. et Roesch L., Mem. Sci. Rev. Metal. LXIX 11 (1972) 815.

[2] Chaboche J. L., Dang VAN K. et Cordier G., SMIRT Conference Berlin, Paper L11/3, W. Germany (1978).

[3] Nouilhas D., Cailletaud G., Policella H., Marquis D., Dufailly J., Lieurade H. P., Ribes A. et Bollinger E., Eng. Fract. Mech. 21 (1985) 887

[4] Challenger K. D. et Moteff J., Metall. Trans. 4 (1973) 749.

[5] Morris D. G., Acta Met. 26 (1978) 1143.

[6] Goodman A. M., et Goodall I. W., Mech. Behaviour and Nuclear Applications of Stainless Steel at Elevated Temperatures, Varese, 20-22 May (1981) Italy, Book 280, Published (1982) by the Metals Society, London, pp. 210-217.

[7] Nouilhas D., Int. Journ. Plasticity 5 (1989) 501.

[8] Contesti E. et Cailletaud G., Proceedings of Mecamat, Besançon August 30 - September 1, France, I. 9-34 (1988).

[9] Delobelle P. et Oytana C., J. Press. Vessel. Techn. Part. I and II 109 (1987) 449.

[10] Matteazzi S., Piatti G. et Boerman D., Mech. Behaviour and Nuclear Applications of Strainless Steel at Elevated Temperatures, Varese, 20-22 May (1981) Italy, Book 280, Published (1982) by the Metals Society, London, pp. 194-202.

[11] BARnBy J. T., J. of Iron and Steel Institute 392 (1965).

[12] Schmidt C. G. et Miller A. K., Res. Mechanica 3 (1981) 109.

[13] BLANC D., Thèse ENSMP, Paris (1986).

[14] Delobelle P. et Lexcellent C., Proceedings of Mecamat, Besançon, August 30, September 1, France, II/135-154 (1988).

[15] Delobelle P. et Varchon D., Rev. Phys. Appl. 18 (1983) 667

[16] Ohno N., Tabahashi Y. et Kuwabara K., J. Eng. Mat. Techn. 111 (1989) 106.

[17] Inoue S. T., Imatami S. et SAhashi T., SMIRT VIII, August 19-23, Brussels, Belgium, vol. L1/2 (1985) 7.

[18] Blanchard P. et Lemoine P., J. Press. Vessel Techn. 110 (1988) 51.

[19] Delobelle P., J. Nucl. Mat. 114 (1989) 364.

[20] Delobelle P. et Oytana C., Nucl. Eng. Design 83 (1984) 333.

[21] Delobelle P., Varchon D. et Oytana C., Techniques for Multiaxial Creep Testing Conference, Cont. Elect. Lab., 25-26 September Leatherhead, England, Chap. V (1985) p. 93.

[22] Solomon A. A., Rev. Sci. Inst. 40 (1969) 1025.

[23] Delobelle P. et Oytana C., SMIRT IX, Lausanne 17-21 August, Suisse, Div. L2/377 (1987).

[24] Delobelle P. et Oytana C., J. Nucl. Mat. 139 (1986) 204.

[25] Cottrell A. H. et Bilby B. A., Proc. Phys. Soc. A 62 (1949) 49.
[26] Friedel J., Dislocations (Pergamon Press) (1964) pp. $410-430$.

[27] Schoek G. et Seeger A., Acta Metall. 7 (1959) 469.

[28] Ficher J. C., Acta Metall., 2 (1954) 9.

[29] Reed-Hill R. E., Park S. C. et Beckerman L. P., Acta Metall. 31 (1983) 10, 1715.

[30] Delobelle P. et Oytana C., Scr. Metall. 12 (1978) 765.

[31] Park S. C., Beckerman L. P. et Reed-Hill R. E., Metall. Trans. 14A (1983) 463.

[32] Lebienvenu M. et Dubois B., J. Phys., Colloq. France 44 (1983) C9-439.

[33] Ke Ting-Sui et YANg Pen-WeI, Sciencia Sinica 6 (1957) 623.

[34] Nowick A. S. et Berry B. S., Anelastic Relaxation in Crystalline Solids (Academic Press) (1972) pp. 330-341.

[35] Billa R. et Delobelle P., IX Brazilian Congress of Mech. Eng., 7-11 Dec. (Florianopolis, Brazil) 2 (1987) 1133.

[36] Billa R., Thèse Besançon, France (1989).

[37] Batz W. et Mehl R., Trans. Am. Inst. Min. Metal. Eng. 188 (1950) 553

[38] Rose K. S. B. et Glover S. G., Acta Metall. 14 (1966) 1505.

[39] Evans J. T. et Douthwaite R. M., Acta Metall. 21 (1973) 49.

[40] Quist O. P. et Carpenter S. H., Acta Metall. 23 (1975) 321.

[41] Delobelle P., Oytana C. et Varchon D., Mat. Sci. Eng. 29 (1977) 261.

[42] Delobelle P., Oytana C. et Varchon D., Mém. Sci. Rev. Métall. 75 (1978) 3, 175.

[43] Rosinger H. E., Graig G. B. et Bratina W. J., Mat. Sci. Eng. 5 (1969/70) 163.

[44] Rosinger H. E., Met. Sci. 9 (1975) 1.

[45] Beckerman L. P., Ph. D. Dissertation, Univ. Florida, Gainesville, USA (1980).

[46] Cribb W. R. et Reed-Hill R. E., Metall. Trans. 8A (1977) 71.

[47] Delobelle P., Oytana C., Varchon D., Metal. Trans. 16A (1985) 361.

[48] Thorpe W. R., et Smith I. O., Mat. Sci. Eng. 49 (1981) 71.

[49] Kocks U. F., Cook R. E. et Mulford R. A., Acta Metall. 33 (1985) 4, 623.

[50] Halphen B. et Nguyen Q. S., J. Mec. Theor. 14 (1975) $1,39$.

[51] Delobelle P., Revue Phys. Appl. 23 (1988) 1.

[52] Mukherjee A. K., Bird J. E. et Dorn J. E., Trans. ASM 62 (1969) 155.

[53] Spingarn J. R., Barnett D. M. et Nix W. D., Acta Metall. 27 (1979) 1549.

[54] Sherby O. D. et Miller A. K., J. Eng. Mat. Techn. 101 (1979) 387.

[55] Remy L., Pineau A. et Thomas B., Mat. Sci. Eng. 36 (1978) 47.

[56] Armstrong P. J. et Frederick C. O., C.E.G.B., Report RD/B/N, 731 (1966). 
[57] Chaвoche J. L., Thèse d'Etat, Fac. Sci., Paris, France (1978).

[58] Burlet H. et Cailletaud G., Eng. Compt. 3 (1986) 143.

[59] Delobelle P. et Lachat R., Soumis à J. Eng. Mat. Techn.

[60] Ohno N., J. Appl. Mech. 49 (1982) 421.

[61] Снавоснe J. L., Int. Journ. Plasticity 2 (1986) 2, 149.

[62] Miller A. K. et Sherby O. D., Acta Metall. 26 (1978) 289.

[63] Delobelle P., XIX Canadian Fracture Conference, Ottawa (Canada), 29-31 May 1989.
[64] Hong Sun I. G., Mat. Sci. Eng. 76 (1985) 77.

[65] Benallal A. et Marquis D., J. Eng. Mat. Techn. 109 (1987) 326.

[66] Benallal A., Le Gallo P. et Marquis D., Proceedings of MECAMAT, Int. Semin. on the Inelastic Behavior of Solids, Models and Utilization, Besançon 30 Aug.-Sept. 1, V/361 (1988).

[67] Снавосhe J. L., $2^{\text {nd }}$ Int. Conf. on Constitutive Laws for Engineering Mat., University of Arizona, Tucson (USA), January 5-8 (1987).

[68] Nouilhas D., soumis à Res. Mech. (1989). 